\title{
Joan Roís de Corella, Lamentació de Mirra, deNarciso, de Píramus i Tisbe: edizione critica
}

\author{
Annamaria Annicchiarico \\ Università degli Studi Roma Tre \\ annamaria.annicchiarico@uniromaz.it \\ https://orcid.org/oooo-OOO-2-7II3-59IX \\ Received 30/03/20I6; accepted 29/o5/20I6 \\ DOI I0.7203/MCLM.3.8132
}

\section{Joan Roís de Corella, Lamentació de Mirra, de Narciso, de Píramusi Tisbe: a critical edition}

\section{ABstract}

This contribution is a partial anticipation of the integral critical edition of the mythological faules of Joan Roís de Corella (XV century) for Barcino (ENC). It derives from the need to revise the textual situation of the most representative faula of the initial Corellan narrative phase, namely Lamentació de Mirra, de Narciso, de Píramus i Tisbe, and to propose, where necessary, new textual lessons. It consists of an introductory section followed by a new edition of the text with its critical and complementary apparatuses. In the introduction, essential information is at first provided on the manuscripts' tradition and previous editions of the Lamentacions, otherwise known as Lo jardi de amor, with reference to the few existing papers specifically dedicated to it. Then, reflections on the diegetic and semantic coherence of the Corellan narration follow, by inspecting on why the author combines and how he amalgams the three myths, and focusing on the traits of the faula's autonomy from Ovidian (or neo-Ovidian) formulation of the three stories. This new edition of the faula is accompanied by footnotes, where questions related to the constitutio textus are discussed in detail.

KeYwORDS

Medieval Catalan literature; Joan Roís de Corella; mythological narratives; Lamentacions; critical edition; Ovid in the Middle Ages.



Magnificat Cultura i Literatura Medievals 3, 20I6, I-35. http://ojs.uv.es/index.php/MCLM

ISSN 2386-8295 


\section{RIASSUNTO}

Il contributo, che è un’anticipazione parziale dell'edizione critica integrale delle narrazioni mitologiche di Joan Roís de Corella (XV sec.) per Barcino (ENC), nasce dall'esigenza di rivedere la situazione testuale della faula in oggetto (Lamentació de Mirra, de Narciso, de Píramus i Tisbe) e di avanzare, dove necessario, nuove proposte di lettura. Esso consta di due parti, delle quali la prima contiene l'Introduzione, la seconda il Testo con note a piè di pagina, in cui si discutono questioni relative alla constitutio textus, l'Apparato e l'Apparato complementare. Nell'Introduzione si danno informazioni essenziali circa la tradizione manoscritta delle Lamentacions (altrimenti note come Lo jardí de amor) e le edizioni di cui sono state oggetto fino ad oggi; quindi si illustra in breve lo stato degli studi (pochi articoli) dedicati ad esse. Seguono riflessioni sulla coerenza diegetica e semantica del testo; sostanzialmente, ci si chiede perché l'autore combini i tre miti e come li amalgami, e quale sia il ruolo svolto da ciascuna delle tre figure esemplari; quindi, si mettono a fuoco i tratti di autonomia dell'opera rispetto alla formulazione ovidiana (o neo ovidiana) delle tre storie.

PAROLE CHIAVE

Letteratura catalana medievale; Joan Roís de Corella; favole mitologiche; Lamentacions; edizione critica; Ovidio nel medioevo.

Annamaria Annicchiarico. 20I6. 'Joan Roís de Corella, Lamentació de Mirra, de Narciso, de Píramus i Tisbe: edizione critica', Magnificat Cultura i Literatura Medievals, 3: I-35

\section{TABLE OF CONTENTS}

o Dati - 3

I Introduzione - 3

2 Testo -9

3 Apparato - 25

4 Apparato complementare -30

5 Opere citate $-3^{2}$ 


\section{ə*⿻}

\section{oDati}

Il tràdito: trasmesse dal Cançoner de Maians (Biblioteca Universitària de València, ms. 7228, ff.

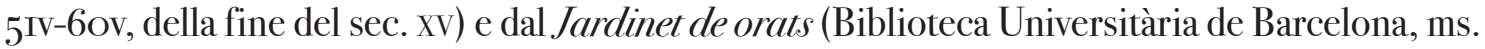
I5I, ff. 64r-78r, la cui stesura fu ultimata il I486), ${ }^{1}$ sono rispettivamente intitolate Lamentaçio de Mirra filla de Cinaras, De Narçiso, De Piramus y Tisbe nel ms. Maians, e Lamentacions de Mirra e Narcisso e Tisbe nel Jardinet de orats, le tre "lamentazioni" d'amore che, in seguito, Miquel i Planas (I9I3) avrebbe designato come Lojardi de amor.

Le edizioni: Miquel i Planas (I9I3: I63-90), riprodotta con ammodernamento ortografico in Carbonell (I973: II5-3I); Martos (2OOIa: I75-98); Escartí (2OI4: 97-II2) che si rifâ, con parziale attualizzazione grafica, al testo delle precedenti edizioni e, fondamentalmente, alla restitutio di Martos (200Ia). Altre: Pelai Briz (I868), Gustà (I980), Almiñana Vallés (1984-I985), Ahuir i López (1997). Per una nuova edizione dell’opera mitologica corelliana, vid. Annicchiarico (2004).

Gli studi: il commento più particolareggiato di cui a tutt'oggi si dispone è la lettura di Martos (2Oorb: 57-IO6), il quale mira soprattutto a rinvenire, e esaminare, gli elementi di continuità e di discontinuità tra il testo corelliano e l'ipotesto ovidiano richiamandosi, anche, ai mitografi tardoantichi e medievali. Con un approccio non dissimile, Wittlin (I997) guarda al Corella traduttorerifacitore di miti e, pur collocando le Lamentacions nella dimensione "d'exercisis escolars, medievals i humanístics", ne mette in valore i tratti che preannunciano il passaggio della scrittura mitografica corelliana "de la traducció a la creació literària". Mentre, nella prospettiva più ampia del nesso tra morale e letteratura, debitamente inquadrato a suo tempo da Badia (I988), osservazioni sulla valenza didattico-esemplare del testo si leggono in Cingolani (I998: I57 ss. e passim). Rilievi sull'articolazione interna dei tre miti componenti le Lamentacions sono in Alemany Ferrer (20I5). Da menzionare, quindi, il commento di alcuni passaggi delle Lamentacions nelle monografie di López Quiles (2007; 20I4) e di López Quiles-Puig Gisbert (2OI4) mirate alla lettura in chiave biblico-filosofica dell'intera opera corelliana. Alla stessa prospettiva interpretativa è riconducibile la lettura del mito di Mirra proposta da Fàbrega Escatllar (2004): la impia virgo ovidiana a confronto con quella che è la vittima, a causa di una "enamorada pietat", di un errore nell'individuazione del sobiran bé. Le Lamentacions, insieme alla Istòria de Leànder y Hero, sono oggetto di una edizione ridotta, a cura di Annicchiarico, con introduzione, commento, note, e traduzione in italiano, di prossima consegna alle stampe. Nell'ambito degli studi, assai fecondi di risultati nell'ultimo cinquantennio, centrati sul riuso dell'opera corelliana nel Tirant lo Blanch, ricordo, per le Lamentacions in particolare, i contributi di Miralles (1978; 199I), nonché quelli di Pujol (2002) e di Hauf (2005).

\section{Introduzione}

Lecosis cosiddette Lamentacions sono una delle nove narrazioni mitologiche composte da Joan Roís Corella, la cui opera, mitologica appunto, rappresenta uno dei momenti più significativi della ricezione di Ovidio nel contesto tardo-medievale catalano, o più in generale iberico, insieme alla traduzione delle Heroides di Guillem Nicolau (Pujol 2OI2; 2OI4) e alla versione delle Metamorfosi di 
Francesc Alegre (Badia 1993; Bescós Prat 2014).

Nell'impossibilità di collocare i singoli testimoni dell'intero lascito corelliano in una successione cronologica più o meno circostanziata, alcuni studiosi (Chiner Gimeno 2OI4; Soler 2OI4), sul fondamento di una documentazione d'archivio da loro stessi portata alla luce e/o sistematizzata in un quadro organico, hanno ribadito l'opinione da tempo corrente secondo la quale il periodo in cui l'autore conseguì il titolo di mestre en sacra Teologia, ovvero gli anni i468-69, rappresenta un discrimine tra un primo e un secondo tempo del suo percorso creativo:

Serà en l'època anterior a l'obtenció del títol de mestre en sacra teologia on cal, en la nostra opinió, situar la major part de l'obra no religiosa de Corella, la denominada obra profana, per tal que la producció literària corellana tinga coherència amb l'experiència vital i anímica del cavaller i mestre en teologia Joan Roís de Corella (Chiner Gimeno 20I4: I63-64).

Fermo restando il convincimento - radicato da decenni nella critica (Riquer I964; Badia I988; Cingolani 1997; 1998; Martos ed. 200Ia; Martos 200Ib; López Quiles 2007) e ribadito più volte ben di recente (Martínez 2OII; 2OI3; López Quiles 2OI4) - che nel percorso esistenziale ed intellettuale di Corella

... no ha de fer-se de la data d'obtenció del títol de mestre en sacra teologia una frontera infranquejable perquè, en la vida de Corella, tampoc no hi ha compartiments estancs - com ja ha estat destacat per Cingolani, Badia o, darrerament, per Martínez Romero (2013) ... (Chiner Gimeno 20I4: I64).

In parallelo, per quanto riguarda la faula in discorso, vale l'idea altrettanto consolidata che la prima fase dell'attività corelliana si articoli in due tappe: una di apprendistato retoricogrammaticale, databile a metà degli anni cinquanta, della quale proprio "les Lamentacions de Mirra, Narciso i Tisbe [...] semblen el fruit més madur" (Gómez 2OI5: 226) e una successiva: "Totes les altres versions clàssiques descriuen un segon moment més prolífic i de plena maduresa ideològica $\mathrm{i}$ artística" che si chiude nei primissimi anni sessanta, cioè nel momento "en què els estudis teològics i la composició de sermons devien començar a monopolitzar la dedicació de l'autor” (ibid. 226).

Come vuole il titolo apposto da Miquel i Planas (I9I3), è esattamente un "inferno-giardino d'innamorati' ${ }^{2}$ quello nel quale sono ambientati i racconti che i protagonisti, Mirra, Narciso e Tisbe, fanno delle loro sofferenze: un adolorit verger in cui un io narrante innamorato scende nella speranza di trovare conforto tra chi prova un dolore simile al suo. In sostanza, un'incursione in un "virgiliano" oltretomba d'amore, dove los devots de Venus sono condannati, più che a scontare il loro peccato terreno, a continuare a soffrire ricordando le rispettive pene d'amore:

Aquí es troba [l'io innamorato] amb tres desgraciats, Mirra, Narcís i Tisbe, els quals - a l'igual de la Dido virgiliana - no pateixen, si són mirats des d'una òptica cristiana, les penes adequades a uns luxuriosos, com és el cas de l'infern dantesc [...]. És aquest un infern tot pagà i virgilià - tot i ser ben barrejat amb el de Dante ... (Cingolani 1998: I84),

e dove la voce narrante "voldria trobar consol, com Ausiàs March -XIII, vv. 5-I2" (Gómez 2OI5:

2. È noto che la tematica degli oltretomba d'amore, in specie gli inferni-giardini d'amore, annovera nella tradizione catalana, tra i secc. XIV-XV, testimoni significativi, come la Glòria d'amor di Bernat Hug de Rocabertí, il Salut d'amor, il lai di Pere Torroella Qui volrà veure un pobre estat. Per un inquadramento di questi testi nel contesto della ficció sentimental catalana del secondo ‘ 40O, vid. la puntuale indagine di Pellisa Prades (2OI3: III-I3 e II7). 
227). ${ }^{3}$

Collocate, dunque, in un verger, la cui descriptio, più che minima, si risolve nell'indicazione di un adolorit verger all'inizio (11. 4-5) e di un trist verger alla fine (1. 538); e introdotte da un io che ascolta e riporta i lamenti, ma la cui voce - e presenza fisica - si esaurisce nelle poche righe del preambolo o dei punti di passaggio da un personaggio all'altro; le tre lamentazioni non sono che la sequenza di tre monologhi che trapassano l'uno nell'altro quasi senza soluzione di continuità, in modo da costituire un unico pianto sulla forza traviante e distruttiva dell'eros.

Insomma, tre lamenti per tre fantasmi, vessilli della fol'amor, responsabili di tre distinte trasgressioni - un incesto, un autoinnamoramento, un'infrazione del codice comportamentale messi in un ordine di comparsa di cui è la stessa Tisbe, ultima dei tre, a dare la ragione, affermando che, rispetto alla sofferenza pur estrema raccontata da Mirra e da Narciso, la sua va aldilà delle parole e della stessa struggente semantica della metamorfosi, in quanto a parlare, per lei, sono i segni concreti della sua sventura: la ferita, il petto trafitto, gli abiti intrisi del sangue di Piramo:

... ne vostres mals tenen rahó de ésser creguts, sinó sols per lo testimoni de vostres paraules. Mas, los meus, sens yo parlar, clarament se manifesten, car està lo meu cor, ensemps ab l'ànima, travessat e les mies vestidures tinctes de la enamorada sanch del meu Píramus. Les vostres nafres són invisibles, e les mies porten ferea de ésser mirades. Los vostres mals demanen piadosos reçitadors, perquè als qui.ls hohiran moguen a misericòrdia; los meus, ells se manifesten e no conssenten ésser hoïts, sinó ab ulls de piadoses làgremes (11. 372-79).

E se gli stessi personaggi già al loro apparire, nel prologo, si affannano (treballant) a recitar la propria pena "cascú en magor grau"; e se Tisbe apre il proprio discorso nel segno del boeziano fuisse felicem ("La major dolor que als mesquins atribula és si en algun temps són estats benaventurats", 11. 369-70) ${ }^{4}$ per affermare che il proprio dolore è al di sopra di ogni paragone; è perché l'ordine di successione è predisposto in modo tale da produrre un crescendo di patetismo e, quindi, di partecipazione emotiva da parte del lettore (Martos 2OOIb: 74; Gómez 2OI5: 228).

Detto ciò, e restando nel passo liminare, non sfuggono all'attenzione alcuni particolari: primo, che a balzare allo sguardo del narratore visitatore appena entrato negli Inferi siano Mirra e Tisbe, senza che si faccia il minimo cenno a Piramo; secondo, e soprattutto, che Narciso appaia insieme a loro, quasi come figura di accompagnamento: "hon viu a Mirra e a Tisbe qui, ensemps ab Narçiso apartats dels altres, de sos mals en fort manera planyien” (1l. 6-7). Particolari significativi, a mio avviso, in quanto prefigurano le dinamiche interne ai ruoli: Narciso, metafora dello specchio riflettente illusioni e fantasmi, nonché cassa di risonanza, tra le due voci femminili, di un eterno pianto sul potere di Amore; e le due dannate, figure della dualità amore incestuoso / amore potenzialmente lecito ma fatalmente segnato dalla 'dismisura'. Altrimenti detto, una voce maschile, eco onniassorbente della coscienza che attraversa se stessa tra luci, ombre, e lacerazioni. E due voci femminili, speculari: Mirra, consapevole dello scelus commesso e condannata a vivere di ricordi ignominiosi; Tisbe, quasi orgogliosa d'aver vissuto un rapporto d'amore in sé non peccaminoso, e,

3. Secondo l'idea che "Cascú requer e vol a son semblant”: “Tot i que Ausiàs March aplica habitualment l'aforisme a l'amor [...] ara se’n serveix per a justificar amb una base científica la mútua repulsió entre el poeta i els altres homes [...] i la seva preferència per la companyia dels damnats" (Gómez-Pujol 2008: I27); vid. anche Bohigas ed. 2005: IO-II. Più in generale sull'aforisma, vid. Tosi (I993: 6оO-оI) e sull'idea, correlata, del Commune naufragium omnibus solatio est, vid. Walther (I982-86) e Tosi (I993: 756-57).

4. De consolatione philosophiae, II.4; il topos, frequentatissimo, ha tra le sue occorrenze più note: Dante, Inf. V, vv. I2I-23; Boccaccio, Elegia di Madonna Fiammetta (Delcorno ed 1994: VIII, 9.1). In ambito catalano, per una ricorrenza ausiasmarquiana del topos, con i relativi riferimenti bibliografici, vid. Gómez-Pujol (2008: 93-95). 
di più, proiettato su un "líçit matrimoni".

Sicché, non è un caso che Tisbe rivendichi con piglio deciso la diversità del proprio vissuto amoroso (11. 397-98), perpetuantesi nelle desiguals penes (1. 538) dell'oltretomba; né sono casuali alcuni particolari che riguardano gli ultimi momenti di vita delle due eroine e il loro destino finale, nella cui raffigurazione si concentra il loro modo diverso di esemplarizzare il rapporto colpa/ castigo.

Non è irrilevante, infatti, che Mirra, preso atto del nefas commesso, chieda di morire, e, inascoltata, venga dagli stessi numi celesti compressa, anima e corpo, nell'albero che stilla lacrime di dolore, a differenza della progenitrice ovidiana che dichiara di non voler offendere con la propria presenza né il mondo dei vivi né il mondo dei morti e implora d'essere trasformata in qualcosa d'altro da sé. ${ }^{5}$

Come dire, all'eroina ovidiana che supplica e ottiene la metamorfosi e, con essa, l'iscrizione in un altro ordine di realtà che non è né la vita né la morte:

“... sed ne uiolem uiuosque superstes

mortuaque extinctos, ambobus pellite regnis

mutataeque mihi uitamque necemque negate".

Numen confessis aliquod patet; ultima certe

uota suos habuere deos ...

(Reed ed. 20I3: Met., X, wv. 485-89),

Corella fa corrispondere una Mirra che subisce, invece, la metamorfosi; ovverosia quell'“aspra penitència", in virtù della quale la "culpable ànima" ottiene lo statuto di anima degli e negli Inferi, nonché il diritto di condividere con pari dignità il destino degli altri due ospiti del jardí:

sopliquí los déus més avant no·m conssentissen viure. Mas, perquè los inferns se entrenyoraven de rebre la mia culpable ànima, no plagué als déus se partís del meu cos fins que fos lavada ab làgrimes de aspra penitènçia, car, puix havia ensutziat la un món, no era rahó ensutziàs l'altre. [...] plagué als déus mudar lo meu cos en arbre de mon propi nom, en lo qual encara-s mostren les làgrimes testificant la mia amargua vida (ll. 196-204).

Più clemenza è invece riservata alla sua Tisbe, cui l'Olimpo concede, infatti, la realizzazione di tutti e due gli ultimi desideri: che il colore cupo delle bacche del gelso resti in eterno la rappresentazione del dolore suo e di Piramo, e che un sepolcro comune ne accolga i corpi:

Mas, perquè la causa de nostra mort per a sempre sia palesa, prech als déus aquest arbre jamés no consenten que lo seu fruyt leixe lo dol ${ }^{6}$ de nostra desaventura. E als déus més soplich, puix en vida a nostres desigs tant han contrastat, ara, aprés mort, ordenen que.ls nostres cossos en un mateix sepulcre reposen (ll. 53'-35).

In altre parole, è come se l'eroina ottenesse un riconoscimento della dignità del suo caso che, per di più, adombra paradossalmente una sorta di avallo divino per quella unione fisica dei due giovani

5. Così Francesc Alegre nelle Transformacions: “mes perque vivint no sia vergonya dels vius ni entrels morts offena las lurs ombres ab la mia presencia lançant me dels dos regnes mudau la mia vida y nom doneu la mort” (I494: X, VI, f. LXXXVIIIII).

6. Da notare che alla lezione prech als déus aquest arbre jamés no consenten que lo seu fruyt leixe lo dol di $V$ corrisponde prech en aquest arbre que james consente quel seu fruyt leix lo dol di $O$ (in linea con l'originale ovidiano); a riguardo, vid. anche n. 7 . 
che proprio dagli dei era stata ostacolata in vita. E ciò, a fronte della stessa Tisbe ovidiana che le medesime invocazioni aveva rivolto rispettivamente all'albero (alla volontà celeste) e ai genitori, vedendo poi realizzati i propri desideri, l’uno dagli dei, l'altro dai genitori:

“.

hoc tamen amborum uerbis estote rogati,

o multum miseri meus illiusque parentes,

ut quos certus amor, quos hora nouissima iunxit,

componi tumulo non inuideatis eodem.

at tu, que ramis arbor miserabile corpus

nunc tegis unius, mox es tectura duorum,

signa tene caedis pullosque et luctibus aptos

semper habe fetus, gemini monimenta cruoris".

(Barchiesi ed. 2007: Met., IV, vv. I54-6I). ${ }^{7}$

Alla Tisbe corelliana, in sostanza, è concessa la facoltà di appellarsi esclusivamente ai numi e di negligere del tutto, in un silenzio risentito, l'auctoritas paterna: quasi che i suoi ultimi istanti di vita ricacciassero nell'indistinta crueldat dels inplacables fats (1l. 52-22) l'intera dimensione familiare, che d'altronde nella memoria non le sopravvive se non in associazione con la cupa immagine del carçre: carçre de nostres mares (1. 383), carçre de la trista casa (1.482), e sotto il segno della crudeltà: crueldat de nostres pares (11. 392-93), cruel paret (1. 4IO).

Queste osservazioni nulla tolgono all'idea che per Corella, predicatore e futuro mestre en teologia, ogni forma di disio carnale sia esecrabile e che per la sua ortodossia morale non possano esserci distinguo nella censura del desordenat voler e di tutto quanto non coincida col sommo bene (inscindibile dall'honestum). ${ }^{8}$ Tuttavia, è indubbio che il Corella auctor e il Corella agens, personaggio-testimone, non guardi con lo stesso occhio i tre interlocutori. Anzi, il testo è costruito in modo tale che al crescente patetismo e alla crescente partecipazione emotiva dell'audience, cui accennavo, corrisponda anche il segno di una diversa complicità affettiva da parte di scrive. Non per nulla Tisbe tiene a esplicitare in che cosa consista la natura ben differente della propria immoderatio, una immoderatio giovanile, rispetto a quella di Mirra, vittima di un errore nella valutazione del bonum, ${ }^{9}$ e di Narciso, vittima del fascino ingannevole delle apparenze:

7. Così Francesc Alegre: "O miserables pares siau pregats [...] vullau posar ensemps en una sepultura y tu arbre[...] te per tostemps senyal ab les mores vermelles de nostra trista mort [...] y estech complaguda sa derrera demanda per los pares y deus durant en lo morer fer les mores tenyides de tan amable sanch y soterrant lurs cossos tots dintre una tomba." (I 494: IV, II, f. XXVIIIIV).

8. Cingolani (I998): “les normes socials de l’honestedat són intransferibles, i no importa, a efectes del tràgic desenllaç, que es vagi contra la voluntat de la família o que s’estimi el propi pare (ı6o) [...] Així que tant en els moralistes clàssics que podia haver llegit o que circulaven per la València del seu temps, com en la seva ètica personal, Corella trobava que qualsevol infracció de l'honestedat era un pecat molt greu” (I63). D’altro canto, è comprensibile che in questo inferno "virgiliano" vi sia da parte del narratore-testimone la sospensione momentanea di “una valoració moral cristiana, que condemnaria sense remissió els luxuriosos amants, e li dóna la facultat de raonar en termes de relativitat moral humana i no d'absolutisme religiós" (I85); analoghe considerazioni in Martos (200Ib: 72-73).

9. Le parole di Mirra ("És natural condició, a la qual fogir és impossible, que nostre voler, sinó en sobiran bé, terme no pot atényer; e si alguna cosa desigam, encara que en estrem sia mala, ab vel ho hombra de bé nostre voler la desiga.", 11. 10-12) rinviano con tutta evidenza al De consolatione philosophiae, III.2.: "Omnis mortalium cura, [...] sed ad unum tamen beatitudinis finem nititur pervenire. Id autem est bonum [...] est enim mentibus hominum veri boni naturaliter inserta cupiditas, sed ad falsa devius error abducit” (Moreschini ed. 20oo: 59-6o). A riguardo, vid. López Quiles (2014: 408, e passim), che riconduce momenti specifici del dettato corelliano ai libri sapienziali, e in specie alla Sapienza e ai Proverbi. Per altre ricorrenze corelliane del concetto espresso da Mirra, vid. nel Debat epistolar entre mossèn Corella i el príncep de Viana: "la voluntat humana, que sempre en lo be pren terme" (Miquel i Planas ed. 
fins tant que atenyguem aquell temps en lo qual poguérem executar lo delitós servir de Venus [...] Emperò, assajam si en líçit matrimoni se poguera fer que, puix nostres ànimes eren juntes, los cossos separats no penassen (1l. 39I-94),

Píramus no era mon pare, com Sínaras de Mirra; ni era la encantada nimfa de Narçiso (ll. 397-98),

lanciando un ben chiaro grido di protesta contro "l'indiscreto" veto genitoriale:

Yo tinch rahó de querellar-me de ma desaventura, car aquells qui follament e sens discreçió donen les veles als vents no.s deuen maravellar si la proa de sos desigs bat los esculls, car a tart de foll navegar se ateny segur passatge. Sols la indiscreta vellea, fallida de memòria, de nostres pares lunyava nostres voluntats concordes (11. 398-402).

Ciò detto, mi sembra che si chiarisca ulteriormente la dinamica interna del trittico. A iniziare dal ruolo che il mito di Narciso, con i suoi elementi costitutivi - la superficie acquatica, lo specchio, il fantasma, il doppio - e con le significazioni che ad esso aveva attribuito la moralisatio medievale - la ricerca/scoperta del sé, il naufragio del sé, l’illusorietà delle apparenze, la caducità dei beni e valori terreni, e così via -, ${ }^{10}$ svolge nella centralità della sua postazione. Nel senso che esso, mentre si istituisce a emblema dell'orgoglio punito dal gran poder de Venus, è al contempo spazio di sovrapposizione ideale tra la solitudine, narcissica appunto, riflessione e quête per eccellenza, e due diversi sguardi interiori: quello pietoso dell'auctor, che condanna e commisera, e quello 'ausiasmarquiano' dell'agens sceso negli Inferi anch'egli come vittima di Amore.

E infatti Narciso, da perfetto doppio, così censura Mirra, ergendosi a difensore delle norme sociali:

Mirra se querella de les leys humanes justament contrastant al desorde de amor desonesta; yo, sol de ma estrema bellea, la qual sovint als enamorats benaventurada fi porta. Però, a mi ha portat en tan gran dan, que per ha dir-ho no tinch bastament de paraules (ll. 2I5-I8),

quindi, affermando orgogliosamente la propria estraneità alla fol'amor:

car de continu lo meu parlar se lunyava de la libidinosa amor de Venus, e la major part de mes rahons endreçava als cans e als falcons en lo servey de la casta deessa (11. 24I-43),

Axí passava ma vida casta, descuydada de aquells treballs que al servir de Venus s'esguarden. E, sovint, per fallits de seny jutjava aquells qui en les flames de amor cremar veÿa. Mas la fortuna, que de continu aguayta als qui en tranquilitat reposen, penssà, ab cautelosos engans, com devers mi se mostraria adverssa (11. 277-8I).

\section{E non senza vagheggiare un'età favolosa:}

Però yo estimava tan poch perdre ni possehir los béns que ella ab si porta, que·ls seus engans en mi poch loch trobaven. E, menyspreant aquelles coses que en estrem als altres jóvens deliten, en los plaents boschs, regats de clares e gentils fonts, prenia sovint posada. E, a la suau armonia de concordants veus de molts e diverssos ocells, aleujava los sotlíçits treballs que la misèria humana

I9I3: I5I, ll. 79-80); e nel Parlament o col-lació: "E, perquè.l treball de la comuna vida humana delit la major part de les sues obres endreça, yo stime gran delit en aquest món attényer no·s dexa, sinó ab ignorància de aquella cosa en què·ns delitam.” (Martos ed. 200Ia: 238, 1l. 24-27). Anche su quest'ultimo passaggio, vid. López Quiles (2OI4: 4I3).

Io. Su Narciso, in questa sede, mi limito a rinviare all’Introduzione di Mancini (ed. 1989) e a Carrega (2OOI). 
porta. E, quasi seguia la condiçió de aquells qui, en la edat primera, ab los pobres e abundants béns de natura passaven lur vida contenta (1l. 282-89).

Di più, non ci sfugge che Narciso e la sua reprobatio si riflettano pienamente nella stessa senefiance allegorico-morale che Corella attribuisce, o attribuirà, alla scelta ("la voluptuosa vida") di Paride ne Lo johí de Paris. ${ }^{11}$ Il pomo donato a Venere, figura di un inferno morale e spirituale che perdura, non per nulla, oltre la morte ("e, fins en los inferns, cercam mortals remeys de nostra amor furiosa") non fa che ribadire l'insormontabile antitesi tra libidine e amore virtuoso, tra militia amoris e caritas:

$\mathrm{O}$, benaventurats en repòs tranquille, aquells qui, desdenyant a Venus, ab castedat matrimonial, ho ab onesta viduytat, ho ab excellent virginitat, de la mar tempestuosa de Venus delliures, en l'onest port segur de pudicícia han lançat ànchores, prenent terra en l'alta ciutat de Paradís (Martos ed. 200Ia: 3І2, 1l. 462-66).

Insomma, l'auctor-agens inserisce il lamento di Narciso, distrutto dall'immagine riflessa di se stesso scambiata per quella di una ninfa, ${ }^{12}$ tra le due voci femminili, non a caso, ma perché Mirra e Tisbe, ciascuna con la sua dignità di vittima vuoi del desordenat voler indotto dal fato, vuoi del fatale connubio di amore-passione e fortuna avversa, si pongano come exempla morali caricati di un ulteriore senso proprio dal cammeo centrale. Assumendo nel proprio io narrante tutto il peso della colpa della propria rispettiva storia d'amore, non importa se abominevole l'una, potenzialmente legittima l'altra, le eroine si ergono come emblemi della fragilità della condizione umana, e femminile nella fattispecie, di fronte al dispotico eros; e ciò in virtù di un ordine etico - sancito dalla presenza stessa di un Narciso doppio dell'auctor-agens - che riconosce solo nel desiderio del sommo bene la somma felicità.

In conclusione, non è facile dire se il "giardino d'amore" di Corella si collochi nel quadro degli esercizi di scrittura ("exercisis escolars, medievals i humanístics”) sul cui terreno si prepara il passaggio "de la traducció a la creació literària", oppure se, dotato di una sua maturità artistica, esso sia già fuori della fase di passaggio. Certo è che, come si è visto, la fictio si propone in una forma narrativa e con una struttura semantica cui non fa difetto la coerenza interna: tre lamenti esemplari, di tre io esemplari, per dare voce, in un unico lamento, alla voglia di affabulare, una volta di più, l'utopia di un amore mondano e onesto.

\section{Testo}

L'edizione assume come testo-base il ms. Maians $(V)$, ben più corretto e affidabile del Jardinet de orats penalizzato, come è noto da tempo immemorabile, da "la mala caligrafia y la poca escrupolositat de la copia” (Miquel i Planas ı9I3: XXVII; Turró I996; Martos 200Ia, 97-98). La

II. In coerenza con le ipotesi a tutt'oggi formulate circa la successione interna delle opere corelliane, Gómez (2OI5: 225) colloca la composizione de Lo johí de Paris in anni non anteriori al I $45^{8}$ e non posteriori al I 464.

I2. Da ricordare che già nel Lai di Narciso, il protagonista crede, di primo acchito, di vedere nella fonte il volto di una donna: "Ne sai coument nomer te doi: / Se dois estre ninphe apelee / U se tu es duesse u fee" (Thiry-Stassin-Tyssens ed. r976: vv. 676-78). Così, a titolo esemplificativo, anche nel Teseida: “... vide nell'acqua la sua effigie stessa, la quale gli parve sì bella, che, credendo che fosse una giovane che dentro gli fosse, s’innamorò di se medesimo, né mai de su quella si partì ..." (Agostinelli-Coleman ed. 20I5: VI, 6I, I79). E nelle Genealogie deorum gentilium: "Nam die quadam, [...] sitiens se in limpidum reclinavit fontem, et viso ydolo suo, quod ante non viderat, existimans fontis ninpham, repente pulchritudinem probavit et captus est ...” (Zaccaria ed. r998: I, VII, LIX, 802). 
lezione di $V$ viene rispettata finché sia accettabile. Raccolgo nell'Apparato le lezioni di $V$ oggetto di emendamento e le varianti del Jardinet de orats. Va da sé che tra queste ultime ve ne siano alcune più significative delle altre: a monte resta sottinteso quello che, nel corso degli ultimi decenni, si è congetturato circa la presenza nelle copie conservate di possibili varianti d'autore ascrivibili a momenti redazionali distinti (Annicchiarico 1996; 2006: 7I ss.; Miralles I998; Martos 2оOга: 97; Hauf 2005: 784). Le note di carattere ecdotico poste a corredo del testo concernono i casi in cui la constitutio textus richieda uno specifico momento di attenzione. L'Apparato complementare raccoglie varianti in massima parte grafico-fonetiche di $O$.

Seguo fedelmente la grafia del manoscritto base, limitandomi a regolarizzare l'uso di $u-v$ e di $i-j$. Per la separazione delle parole, l'accentazione, l'uso dell'apostrofo e del trattino, mi attengo alle norme ortografiche del catalano moderno, indicando col punt volat le elisioni che oggi non hanno rappresentazione grafica. 


\section{LAMENTACSIÓ DE MIRRA FILLA DE ÇÍNARAS}

Ab desig de trobar a ma dolor semblant companyia, he desemparat aquest món, devallant en los trists e tenebrosos palaus de Plutó, per aquell camí que al desterrat de Troya mostrà la ínclita Sibil-la. E axí só arribat en aquell adolorit

5 verger, en lo qual los devots de Venus, en continu plor, lurs penes reconten; hon viu a Mirra e a Tisbe qui, ensemps ab Narçiso apartats dels altres, de sos mals en fort manera planyien, treballant cascú en magor grau recitar lur pena. Però, primer, Mirra començà en semblants paraules reçitar la sua gran desaventura:

IO "És natural condició, a la qual fogir és impossible, que nostre voler, sinó en sobiran bé, terme no pot atényer; e si alguna cosa desigam, encara que en estrem sia mala, ab vel ho hombra de bé nostre voler la desiga. Axí aparegué a mi ésser cosa piadosa e digna de gran mèrit amar mon pare més que a persona altra alguna. E menyspreant matrimonis de prínçeps e reys, no elegí jamés

I5 partir-me de mon pare, car penssava la sua bellea ésser digna de ma servitut, ab tot que ell sovint me deÿa:

'O Mirra, puix la tua edat de bells néts me fa promesa, digues-me, a qui seràs contenta liurar los teus honrats tàlems'.

Mas, yo callant, per la mia cara de vergonya colorada cahien làgremes

20 semblants a fonts corrents sobre fresches roses. E penssava Sínaras yo ploràs per temor de entrar en la més plaent que perillosa batalla, la qual les honestes donzelles mostren tembre; e, lohant la mia vergonya, ab les sues mans sechava les abundants fonts de mes làgremes. E deÿa que no era maravella la nova terra refusàs lo primer aradre. Demanant-me altra vegada

25 qual me paria digne de possehir ma castedat en líçit matrimoni, responguí: 'Yo desige marit que en res a vós no dessemble'.

O estrema pena no gosar manifestar lo mal a aquell metge de qui sol la salut (52r) s'espera! E, si les paraules deuen ésser semblants a aquella cosa de què parlen, en gran part ne tinch yo fallença per no poder dir la causa de mon

12. nostre voler la desiga: conservo la lezione di $V$, d'accordo con Miquel i Planas (165) e Carbonell (115). Diversa la lettura di Martos (e Escartí, 97) che, sul fondamento della lezione di $O$ (lo tal voler nostre demana), emenda: lo tal nostre voler demana (176; 364, n. 14-15), richiamandosi alle puntualizzazioni di Miralles (1991: 6-8 = 2012: 168-170). Ma, quanto osserva Miralles riguarda esclusivamente la coerenza logico-sintattica dell'intero passaggio del cap. CCIX del Tirant costruito sulle "raons" della Mirra corelliana: "... Si alguna cosa la donzella desija, encara que en strem sia mala, ab vel o ombra de bé lo tal nostre voler demana” (Hauf ed. 2005: 834).

14. de prínçeps e reys: fra gli editori, solo Martos (176), seguito da Escartí (97), accoglie la lezione de grans reys di $O$.

17. dopo promesa: Miquel i Planas (166; Carbonell, 115) e Martos (176; Escartí, 97) integrano e la tua bellesa contrasta als frets vots de diana da $O$.

22. vergonya: casta vergonya con integrazione da $O$ da parte di Miquel i Planas (166; Carbonell, 115) e di Martos (176; Escartí, 97).

23. abundants: sues abundants V; d'accordo con Miquel i Planas (I66; Carbonell, II5), e diversamente da Martos (I76; Escartí, 98), espungo sues, probabile svista per memorizzazione del precedente sues.

25. possehir: fra gli editori, solo Martos (176; Escartí, 98) accoglie la lezione levar di $O$. 
30 mal, car no sé si amor ho infernal fúria havia nafrat lo meu ànimo, obligantme a tant desordenat voler, al qual no podent resistir, en mi deỹa:

'O Mirra, e hon esperes lo terme de tos desigs? O Déus immortals! Resistiu ho mudau lo meu culpable voler, si culpa en si porta'.

Però, recort-me dels animals, los quals obliden qualsevol deute de

35 parentesch; se ajusten per multiplicar son linatge; ne a la egua par mal que son pare sia avi e pare de sos fills, e sovint l'oçell conçep de aquell qui l'à conçebut. Mas, la celçitut humana, enemigua y envejosa del meu delit, ha trobat leys cruels, enemigues de ma enamorada pietat, les quals defenen lo que natura líberament atorgua. E més, he hoït dir en una part del món habitar

40 gents que no han vergonya que una sola sia mare e germana de sos fills. D’on veig clarament lo meu desig no ésser del tot mal; car les coses que en estrem són males en algun temps ni en alguna part poden ésser bones, ni les leys poden ésser fetes en perjuhí del gran poder de Venus, lo qual sobre los déus en pus alt grau trihunfa.

45 Mas, per què vaig en vanes paraules, sinó que desempare la pròpia terra, ans que en mon delliber tanta erra caygua? E, perdent la vista de mon pare, atenyeré conort ho mort desesperada, venjadora de mon peccat. Mas contrasta’m lo delit, lo qual algú no·m pot toldre, car Sínaras és content de piadosament besar-me, e no $\cdot$ s enuja que jo repose entre los seus bells braços.

$5^{\mathrm{O}} \mathrm{E}$, si algun càrrech ne reporte, pensse que les coses de gran estima no sens gran treball se deixen atényer: per ço fon Júpiter quasi patriçida per senyorejar lo çel. Mas les nostres erres paren majors perquè-ls déus ab lo gran trihunfo de lur poder cobren la legea de ses culpes'.

E mentres tals contrasts ma enamorada penssa ab eguals forçes combatien,

55 la nit ab tenebroses ales occupava la terra e, amonestant plaent dormir als animals, atorgà repòs als cossos e a les fatigades pensses. Mas tal repòs yo no aconseguia, car los forts penssaments major força en la callada nit $\left(5^{2 v}\right)$ prenen e a les enamorades pensses ab més poder combaten en la sotliçitut tenebrosa. Axí, en tal temps ma dolor aumentava; e, quasi fora de seny, entrí

6o en la mia cambra fengint que terrible son fatigava la mia trista persona perquè-m lexassen estar sola, car tota companyia me era fort enujosa, puix no podia atényer lo que tant desigava. E, lançant lo meu cos, per fexugua càrregua pesat, sobre.1 meu lit, ab tan desiguals enugs no sabia qual millor que mort elegís. E penssí que, lexant-me de viure, feỹa ma culpa palesa e

65 aconseguia perpètua infâmia; e fóra millor assajar en vida si tal infâmia me

34. obliden: oblidant, emendano Miquel i Planas (I66; Carbonell, iı6) e Martos (I77; Escartí, 98), sulla scorta di O: oblidant se de qual se vol.

36. de aquell qui l'à: non motivato l'emendamento de aquell qui és concebut, compiuto in parte sulla lezione de qui es di $O$, di Martos (177; Escartí, 98).

57. callada: è la lezione, corretta, di $O$, già accolta dai precedenti editori, a fronte di calda di $V$.

59. aumentava; e, quasi: i precedenti editori accolgono la lezione tant augmentava que quasi di $O$.

63-64. enugs no sabia qual millor que mort elegís. E: poiché millor può sottintendere senza difficoltà "enug", cade la necessità, già avvertita da Miquel i Planas (seguito dagli altri editori), di ricostruire il testo sulla scorta di $O$ (... qual part millor ... elegis mes): enug[s], no sabia qual part millor que mort elegis; mes (Miquel i Planas, 168). 
portaria algun delitós guardó. Encara me paria fort gran càrrech que, ans de haver errat, fos de mi omecida.

O envejós enemich, aquell que de continu combat sens vencre ni ésser vençut! Ni la legea de mon peccat me podia retraure de no amar ab tant

70 desordenat voler, ni tampoch la granea de ma benvolença bastava que clarament elegís amar lo que tant desigava. Mas, a la fi, yo viu espedient com mos mals sempre remeyarien, apartant l'ànima del cos per qui totes passions vénen, recorrent a.quella certa infançonia de la mort, la qual egualment accepta qualsevol que a ella recorre. E ligant-me lo coll ab la corda de la 75 cortina, lexí caure lo meu ja quasi fret cos del lit, dient ab veu fort alterada:

'Vixqua Sínaras, puix mor per ell Mirra!'.

Mas los cruels fats, envejosos de ma casta ignoçènçia, tant honest morir no $m$ consentiren. Ans portaren lo murmur de mes darreres paraules a les fels orelles de ma dida, manifestant-li quant ma vida perillava. O vellea, freda

80 enemigua de ma fama, que no conssentint dormir ferm aquella sola hora la qual podia restaurar ma vida! Hoynt donchs l'antigua dona lo temerós so de mes darreres paraules, que manifestava la dolor d'aquell d'on venia, no pereosament se levà del seu lit, car lo desig que de acórrer-me tenia li portava novella força; $\mathrm{e}$ ab tremoloses mans deslliguà aquell las que de mos desigs,

85 ensemps ab ma vida, era terme, acçeptant ma persona (53r) en aquella falda en la qual, en ma primera edat, moltes vegades reposat havia. E, puix que.l cos hagué cobrat sos primers sentiments, ab veu tremolant per temor tant com per vellea, dix semblants paraules:

'O més cruel que tigre, més superba que leona! Si de tu no has piatat, com 90 te doldràs dels estranys? Si a la tua bellea no perdones, qui trobarà en tu misericòrdia? E si de dos mals lo menor és de elegir, qual serà l'altre, si la mort per menor elegexes?'

Mas yo sospite que tu tems en esdevenidor alguna infâmia, e creus, morint, ésser-ne delliure. No.t recordes que major fon la erra de la romana Lucrèçia

95 quant se matà, que si voluntàriament hagués conssentit al desordenat voler de Tarquí? E més si era ignoçenta de culpa, car les justes leys sols als molt culpables obliguen a mort. Quina aygua, per sobresclara que sia, lavarà les tues mans, si són tinctes de sanch ignoçent? Mas, puix lo remey de la mort, del qual est çerta no·t fallirà, tens, penssa si per algun espedient los teus tant

IOO forts mals descanssar porien.

E no duptes dir-me la dolor que la tua ànima tant atribula, puix est segura sinó al que tu voldràs no la faré palesa; e, encara que acabat remey no esperes, attenyeràs algun descans, car delit és als tribulats si lur passió a persona fel recontar poden. Però tu no·t desesperes del meu soccós per la IO5 mia pereosa vellea, car de gran estima són les forçes de l’ànima, les quals, ab doble poder, en los vells reposen. E, si algú te ha ofesa, tens poder absolut en

72. l'ànima del cos per qui totes passions: da segnalare, benché incrongruente nel contesto, la sorprendente lezione la mia anima del noble cors per qui totes potencies addotta da $O$.

102. acabat: de mi acabat è la lezione di $O$ accolta da Miquel i Planas (170; Carbonell, 118) e da Martos (180; Escartí, 100). 
lo regne de ton pare, ab lo qual pots punir al qui-t parrà ésser culpable, ho, si deixes a mi aquest càrrech, sé yo la virtut de les erbes e l'art de nigromànçia, ab les quals coses amagadament te faré venjança. E si pensses contra tu los

IIO déus sien yrats, tenyierem lurs ares de sanch de honrats e placables sacrifiçis, car en nostre poder està amanssar la lur ira, puix perdonar és lur (53v) ofiçi. $\mathrm{Si} \cdot \mathrm{t}$ dols per pèrdua ho abssènçia de alguna persona a qui en estrem ames, recor-te quant mal reparà Dido matant-se. No pensses en los mals abssents sinó com en les coses passades, de les quals hom se deu servir per sol

II5 exemple dels esdevenidors.

Alegra’t ab la tua estrema bellea, la qual, si vols, te farà possehidora de no poch gentil enamorat. E no duptes les plaents e no mortals ferides de Venus per guardar la flor de ta casta corona al qui s'espera ésser ton marit. Fia en ma gran astúçia e elegeix lo que plasent te sia. E, si vols acabat desig atényer, no

I2O pensses los perills esdevenidors, car pochs aradres sofiriria la nova terra si los lauradors lo mal any temien.

Si de fortuna te querelles, no la veig contra tu adverssa, car està en prosperitat lo teu regne e viu ton pare honrat de honesta muller'.

Hoyint yo aquest mot de 'pare', tolent-me plor e vergonya la paraula, no·m

I25 poguí retraure de un tan gran sospir, que penssà la mia dida que, essent yo molt enamorada, temia castich e reprensió de pare. Ab les mans fredes exugant la mia humida cara de moltes làgremes, demanà'm altra vegada la causa de ma dolor, prometent-me dar remey a ma pena. E, ab paraules dignes de fe, me feÿa segura no u sabria mon pare. O cruel seguretat, que $\cdot \mathrm{m}$

I30 tolia lo perill en lo qual sol ma vida era segura! E, lançant-se davant los meus peus, jurà li digués la causa per hon ma vida tenia avorrida.

Yo fóra alegra ella sospitàs mon mal, sens que mes paraules no u declarassen. Mas, qui sospitara lo que tant a tart s'esdevé? Però, a la fi per dar loch a la inportunitat de tan animosa vella, ab sobresgran pena, la mia

I35 lengua formà tals paraules:

'O dona enemigua de honestat! Per què promets altre remey que mort a ma dolor? Tu desiges saber mon mal, lo qual no conssent ésser parlat, perquè l'ayre s'entrenyora $(54 \mathrm{r})$ de rebre lo so de tals paraules e les tues orelles haurien ferea de hoyr lo desorde de tanta amor. Sens comparació és a mi I4O millor morir ab honesta fama, que, vivint, morir de eterna infâmia.

Demanes-me de qui tinch querella: sols de les leys humanes, les quals, ab gran enveja, me lunyen de aquell a qui natura, ab molt estret grau, me acosta. Vols saber ab gran pressa què desige? Sies çerta persona al món benaventurada sia no estime, sinó sola ma mare, que té Sínaras per marit’.

115. dels esdevenidors: non è da escludere una concordanza ad sensum con mals; viceversa i precedenti editori - Miquel i Planas (170; Carbonell, 118), Martos (180; Escartí, 100) emendano de les esdevenidores su $O$ (de les sdevenidores).

119. desig: i precedenti editori - Miquel i Planas (I7I; Carbonell, II8), Martos (I80; Escartí, IOo) - optano per delit, lezione di $O$.

126. Les mans fredes: les sues mans fredes adducono Miquel i Planas (171; Carbonell, 118) e Martos (181; Escartí, 100), sulla scorta di les sues fredes mans di $O$.

128. paraules: juraments $O$, lezione accolta da Martos (181; Escartí, 101). 
I45 E parlar més avant no·m conssentí vergonya. Mas ab dolorosos sospirs manifestava lo cubert seny de mes paraules, tant que entés la sàvia dida lo gran desorde de ma benvolença, e ab suaus paraules assajava si de tant desllimitat desig me poguera retraure. Però, puix véu que lo foch de mon voler, resistint, més aumentava, penssà ab novella esperança si poria

I5O restaurar ma vida, dient:

'No dubtes, Mirra, ni.t desesperes per la dificultat de ton desig, car tu hauràs lo teu Sínaras'. Hagué ferea de dir pare.

Penedint-se ja los cruels fats de tant haver alargat ma vida, eren sotlíçits com en desonrada mort de mi pendrien venjança. E acostumaven en lo regne

I55 de mon pare çelebrar una gran festa de aquella deessa, [...] es, en virtut de la qual la terra fructificava. E les nostres matrones, ab corona d'espigues, ornaven les ares de novelles ofertes. E, per fer més acceptables lurs sacrifiçis, apartant-se de la religió de Venus, leixaven per nou dies los lits frets a sos marits. Paregué bé a la avisada vella en aquest temps enganar mon pare, fentI6o li present de una no poch gentil donzella, dient:

'No·t desplàçia, Sínaras, acceptar en lo teu lit la donzella de qui yo·t parle, car no és menys bella que la tua Mirra'.

Descanssaven ja los cavalls de Febo en los humits palaus del gran Ocçeano, e les esteles corrien als frets banys de Tethis per deixar la calor que de Apol.lo

I65 havien presa. E quasi tot lo món en general sçilençi reposava, ecçeptats los sotlíçits galls, qui, ab altes veus, de la miganit portaven embaixada. En tal hora, ab gran desig, Sínaras esperava la tant lohada donzella.

Yo viu Diana perdre la lum sens que la terra no la y tolia e sentí ab gran (54v) murmur, querellar la terra, enujada de sostenir lo meu abominable

I7O pecat. Mas la certa esperança del esdevenidor delit me feÿa oblidar la granea de tan leig delicte, que qualsevol dolor que de tanta erra esperàs, me paria poca en satisfacçió de tanta glòria.

Pres-me per la mà la esforçada vella, la qual yo seguí com a tremuntana ferma de mon viatge. E, entrant primera en la cambra de Sínaras, ab veu molt I75 alegra, li dix:

'Presta està la tua donzella. Aparta la lum, car la castedat li porta temerosa vergonya e los novells orts de Venus no poch temen lo primer cultivador'.

Hobeý Sínaras les paraules de la vella. E les tenebres, robadores de vergonya, me portaren esforç de entrar en la escura cambra, tenyint de verge I80 sanch aquells tàlems en los quals fuy conçebuda. E, tremolant en los braços

145. dopo vergonya: la qual, sovint de extrema amor es inimiga, lezione di $O$ integrata concordemente dagli editori, a partire da Miquel i Planas (172).

155. s'intenda Ceres, secondo la condivisibilissima congettura di Miquel i Planas (172) che considera la lezione ço és di $V$ (omessa in $O$ ) una corruttela di Ceres, appunto: ne accettano l'emendamento anche i restanti editori (Martos, 182; Escartí, 101), tranne Carbonell che omette la lezione (119).

169-170. sostenir lo meu abominable pecat. Mas la certa esperança ...: da segnalare, in merito alla lezione sostenir lo meu abominable cors mes certa speransa... addotta da $O$, ossia in merito alla variante cors, che peraltro è meno valida nel contesto di pecat, un passaggio “corelliano" del Tirant lo Blanch, cap. CCVIII (mio il corsivo): “... sostenir e comportar lo meu aflegit cors. Mas, la certa sperança que del sdevenidor delit tenia, me feÿa oblidar la granea que amor en si porta. E..” (Hauf ed. 2005: 832, 833, n. 3). 
de Sínaras, sovent, esguardant ma poca edat, me deÿa 'filla'; e ab suaus paraules, apartava de mi tota temor, dient:

'No duptes soferir un mal tan poch perillós, per tants esdevenidors delits'.

Però, ans que la aurora de la venguda de Febo portàs embaixada, quasi

I85 contra voluntat de Sínaras, me partí de la cambra, portant dins mi un fill e germà, nét e fill de mon pare. E, ab tot que mon voler hagués descanssat en lo terme que tant desigava, no·m consentí que per tant poch descans fos contenta. Ans, tornant moltes vegades en la legea de mon peccat, desigà Sínaras saber qui era la donzella que tant semblava a Mirra; e, ab lum I9o enemigua dels enamorats engans, descobrí la culpa de ma benvolença.

E, quasi esbalahit de tant gran erra, falliren-li paraules per a rependre'm de tan gran crim. E, ab cuytats passos, corregué a la espasa que prop si tenia, e asajà tolre'm la vida. Mas plagué als piadosos déus donar-me esforç de tant fogir, que no tingué poder Sínaras de ensutziar les mans en pròpia sanch.

I95 E, puix la sperança de mon delit tenguí del tot perduda, conexent la granea de ma culpa, sopliquí los déus més avant no·m conssentissen viure. Mas, perquè los inferns se entrenyoraven de rebre la mia culpable ànima, no plagué als déus se partís del meu cos fins que fos lavada ab làgrimes de aspra penitènçia, car, $\left(55^{r}\right)$ puix havia ensutziat la un món, no era rahó ensutziàs

200 l'altre. E, perquè les altres dones, hohint la legea de ma benvolença, no creguessen la major part ésser faula ho, almenys, prenguessen esmena mirant la legea de mon peccat, plagué als déus mudar lo meu cos en arbre de mon propi nom, en lo qual encara·s mostren les làgrimes testificant la mia amargua vida".

\section{DE NARÇISO}

No sé si més avant parlà Mirra, ho si en tals paraules se deixà de recontar la desaventurada fi de sa vida; mas, viu Narçiso alçar los ulls, los quals de continu endreça a la amargua font que de les làgrimes de Biblis abundantment corria. E, quasi interrompent lo plant de Mirra, ab dolorosos 2IO sospirs començà de sos mals en semblant manera plànyer:

"Puix als cruels fats plau encara aprés nostra mort los desiguals enugs de amor nos atribulen, sens que altre major descans no atenyem sinó recontar nostres passats mals, escolten tots aquells qui en lo temple de Cupido devotament adoren. Digua cascú si semblant dolor a la mia jamés ha sofert.

2I5 Mirra se querella de les leys humanes justament contrastant al desorde de

202. legea: conservo la lezione di V d'accordo con Miquel i Planas (I74; e Carbonell, I2I) e diversamente da Martos (I84; 373, n. 234) e Escartí (IO3) che optano per justa penitencia di $O$ (non senza una motivazione plausibile: "El que es mira per a aprendre és la penitència que fan del seu pecat"). Se non è da escludere, infatti, che legea sia una svista dovuta alla memorizzazione di legea de mon peccat (1. 188) e/o di legea de ma benvolença (1. 200), neppure è da negare l'effetto intensificante del parallelismo legea de ma benvolença / legea de mon peccat.

203-204. les làgrimes testificant la mia amargua vida, si attengono ad $O$ solo Martos e Escartí: les mies amargues làgrimes, testifficant la mia desaventurada vida (rispettivamente I84 e IO3).

212. altre major descans: altre major delit adducono Martos (185) e Escartí (103), seguendo parzialmente $O$ (altre delit). 
amor desonesta; yo, sol de ma estrema bellea, la qual sovint als enamorats benaventurada fi porta. Però, a mi ha portat en tan gran dan, que per ha dirho no tinch bastament de paraules (55v).

Partint-me de aquella poch anssiosa edat de adolesçènçia, la mia bellea 220 tant aumentava, que la bella reyna Juno més de mi que del gran Júpiter fóra contenta. Mas yo, curant poch de obehir a Venus, en la apartada religió de Diana, ab gran supèrbia me defenia de les enamorades fleixes de Cupido. E, ab lo plaent treball de la caça, alleujava los grans enugs que la vida humana porta. O! Quant sovint les nimfes per contemplar a mi deixaven les clares

225 fonts e, menyspreant lo servir de Diana, malahien la mia bellea, que de tanta supèrbia era acompanyada!

Però, més que totes de mi-s querellava aquella desaventurada nimfa Equo, la qual no tenia poder de parlar sinó les darreres paraules que hohia. L'altre parlar li havia tolt Juno, perquè tenia per costum Equo, ab gentils paraules,

230 detenir la gran deessa quant Júpiter, en delits furtats, ab les belles nimfes reposava; e axí eren cuberts a Juno los amagats plaers de Júpiter. Vehent, donchs, la poderosa reyna que per lo gentil parlar de Equo havien loch de Júpiter los adulteris, penssà com, ab justa penitènçia, d'ella prenguera venja. E, puix sos engans havien fi ab moltitut de gentils paraules, volgué que, d'aquí

235 avant, ne tingués tanta fallença que sol pogués dir lo darrer so de aquelles paraules que a les sues orelles arribarien.

Aquesta era la nimfa la qual, entre les altres, de continu foch d'estrema amor cremava, e més perquè no podia dir quant la mia bellea la enamorava. Y, encara que moltes vegades, seguint ella son costum, digués aquelles

240 mateixes paraules que de mi hohia, ja per axò no manifestava la granea de sa benvolença, car de continu lo meu parlar se lunyava de la libidinosa amor de Venus, e la major part de mes rahons endreçava als cans e als falcons en lo servey de la casta deessa.

Axí passava la trista nimfa lo temps de sa penada vida, esperant quant

245 parlaria yo alguna cosa a la qual ella responent se pogués la sua dolor fer palesa, sens que altre delit no atenyia sinó sol esguardar la mia estrema bellea. Tant que un jorn, fatigat del treball de la cassa, e més de la enujosa calor de Apol·lo, reposant (56r) en la plaent y escura ombra de un vert lorer, ab altes veus diguí:

250 'O gran Febo, espill en lo qual totes les creatures se miren! Si la tua calor enug no portava, estrema és la tua bellea'.

Envides la mia lengua formà tals paraules, quant respòs Equo:

'La tua bellea', que de més dir no tenia liçènçia.

E, tornant yo en les mateixes paraules de Apol·lo, diguí altra vegada:

255 'Sovint, la tua calor estrema pena porta'.

Mas la enamorada nimfa, deixant la major part de mes paraules, solament dix:

242-243. en lo servey: e en lo honest servey $O$, lezione accolta da Miquel i Planas (I77; Carbonell, I22) e da Martos (I86; Escartí, IO4).

247-248. enujosa calor: Martos (186; Escartí, 104) adduce extrema enujosa calor, rifacendosi alla lezione extrema calor anuyosa di $O$. 
'Estrema pena porta'.

Però, les sues respostes no trobaven en mi loch de ésser enteses, que yo

26o esperava si aquell suau vent Zèfiro daria algun socós a la mia fatigada persona, al qual, tornant, deÿa:

'O suau Zèfiro!, puix tens per costum de gran calor fer-me delliure, ara, per què fuigs de mi?’

E no tardà la bella nimfa respondre, ab veu piadosa dient:

265 'Per què fuigs de mi?'

Mas lo foch de gran amor que dins si cremava, no li comportà més avant esperar. Ans asajà, venint a mi, lançar los seus bells braços sobre.1 meu coll, de la qual yo, fogint, ab irada veu diguí:

'Pert de mi la sperança, graçiosa nimfa, que may seré ab tu mentres vixcha'.

$27 \mathrm{O}$ E no.s oblidà ab gest fort adolorit respondre:

'Seré ab tu mentres vixcha'.

Mas, puix véu de son voler la esperança del tot perduda, entrant en les escures silves, en plànyer e plorar despenia tot lo temps de la sua dolorosa vida. Tant, que amagrí la sua gentil persona, que la trista ànima abitar en tant

275 dèbil cos més avant no soferí; los ossos de la qual, convertits en dures pedres, sempre responen, seguint lo costum de aquella de qui eren.

Axí passava ma vida casta, descuydada de aquells treballs que al servir de Venus s'esguarden. E, sovint, per fallits de seny jutjava aquells qui en les flames de amor cremar veÿa. Mas la fortuna, que de continu aguayta als qui

280 en tranquilitat reposen, penssà, ab cautelosos engans, com devers mi se mostraria adverssa.

Però yo estimava tan poch perdre ni possehir (56v) los béns que ella ab si porta, que.ls seus engans en mi poch loch trobaven. E, menyspreant aquelles coses que en estrem als altres jóvens deliten, en los plaents boschs, regats de

285 clares e gentils fonts, prenia sovint posada. E, a la suau armonia de concordants veus de molts e diverssos ocells, aleujava los sotlíçits treballs que la misèria humana porta. E, quasi seguia la condiçió de aquells qui, en la edat primera, ab los pobres e abundants béns de natura passaven lur vida contenta. Fins tant que, un dia, enujat de la estrema calor de Febo,

290 desemparant lo servir de la casta deessa, entrí per una vall, la qual molts arbres axí çircuhien, que no comportaven en algun temps se calfâs de les grans flames de Apol.lo. E davall los arbres estava la terra, de moltes colors quasi brodada ab diverssitat de florides erbes, les quals regava una clara e bella font que, per mig de la silva, ab suau e gentil so corria, que plaent dormir

295 amonestava als qui hohien les veus de tal aygua, la humiditat de la qual a les erbes, que prop li estaven, de color vert sempre vestia.

Fuy no poch alegre acçeptar repòs en tant delitosa posada; mas, per deixar la set que del gran treball dins mi portava, tocant ab los genolls la florida erba, assagí beure en la font de tant gentil aygua, dins la qual viu una tal nimfa, que

300 seria fallit d'enteniment qui en sa presènçia algun altra lohàs per tan bella. E, maravellat per l'estrem de tanta bellea, la primera set tenguí perduda, sens que no tenia poder apartar los ulls de tant delitosa vista. Ne menys tenia

274. amagrí: amagrí tant legge Martos (เ87; Escartí, I05), integrando da $O$. 
atreviment banyar les mans en la freda aygua per tocar la tan graçiosa nimfa, que més que a Júpiter temia fer-li ofenssa. Mas, esperava hoyr d'ella tals

305 paraules que.m manifestassen l'estament de sa vida, que a mi paria que ella, no menys en mi que yo en ella, contemplava. Però l'estrem grat que de la sua bellea tenia, causà dins mi, sens larguea de temps, tanta benvolença que $\cdot \mathrm{m}$ forçà yo primer, en semblant estil, li digués a quanta amor la sua bellea novament me obligava.

3IO $\quad(57 \mathrm{r})$ 'O , més bella que Diana! Quant tinch agrahir a Febo que, per fogir a la sua calor enujosa, sia arribat en aquest tant plaent bosch, en lo qual só fet merexedor de contemplar la tua estrema bellea! Ara só no poch content que haja menyspreat les altre nimfes, perquè.l meu servir en major grat acçeptes, que més me obliga la tua gran vàlua que les homils pregàries de les altres,

3I5 sens que no tinch poder de satisfer los mèrits d'elles, ni poch amar a tu, encara que jamés no·n esperàs guardó; que la bellea de Narçiso, sinó de la tua sola, mereix ésser possehida. Hix de la clara font, car perjuý és a la tua bella cara que per mitjà algú sia vista; si de mi tems ofenssa, asegure’t la tua bellea en la qual yo adore, com a Déu de qui espere infinida glòria. E sies çerta sols

320 en plaure a tu só alegre largament despendre lo temps de ma enamorada vida'.

De tant enamorades paraules esperava yo resposta no gens duptosa, que lo seu gest mostrava no enugar-se del que mes paraules rahonaven. Aquesta era la major dolor que ma penssa soferia, que no sé qual desaventura nostres

325 voluntats concordes separava: yo rrient, ella.s mostrava riure; yo plorant, ella plorava tant que per la moltitut de ses lagrèmes l'aygua de la clara font aumentava.

O cruels fats, los quals no consentien les sues paraules a les mies orelles arribassen! No sé si la remor dels arbres ho tolia, que, yo parlant, ella parlava, e 330 ans responia que mes paraules no acabassen. E jamés lo so de la sua delicada veu poguí perçebre. O quantes vegades assagí ab la mia boca tocar la cruel aygua! No per beure, que la primera set tenguí perduda, mas per besar la gentil deessa, puix no li mostrava desplaure. Ans se acostava a mi tant que ab la cara tocava la enemigua font; e, lavors, se mostrava ella tan fort

310. tinch agrahir: non richiesto dalla sintassi l'emendamento tinch que agrahir fatto da Martos (189; Escartí, 106) su tench que grahir di $O$.

317-318. font, car perjuý [...] algú sia: manca in V; i precedenti editori (Miquel i Planas, I80; Carbonell, I24; Martos, I89; Escartí, Io6) integrano da $O$ (font car perjuy es a la tua bella cara que per metge algu sia), trascrivendo metge tale e quale e, comunque, non chiarendo come l'integrazione dia senso compiuto al passaggio. Viceversa, l'emendamento di metge (possibile corruttela per mitja/metja) in mitjà, inteso come mezzo (in questo caso acquatico), assicura il senso.

318. asegure't la tua bellea: concordo con Martos (189; Escartí, 106) nel conservare la lezione di $V$; diversamente da Miquel i Planas (180; Carbonell, 124) che accoglie la lezione di $O$ : asseguret en la tua bellesa.

319. en la qual yo adore: a la qual yo adore $O$, lezione accolta dai precedenti editori; conservo la lezione di $V$, in considerazione anche della ricorrenza dello stesso sintagma nel Corella lirico (Del jorn que us viu d'altra gens no em pot plaure): ab ferm voler ma pensa en vós adora (Carbonell, 61; Verger 2011: 58; Escartí, 332).

326. ses: solo Martos (189; Escartí, 106) opta per les, lezione di $O$. 
335 enujada, que feÿa continença fogir de la mia vista. Mas, puix me veÿa retraure del que li era ofenssa, reposava en la seguretat primera.

O estrema (57v) pena veure's prop del que és inpossible atényer. O cruel dona aquella que us promet lo que jamés no us dóna, fent-vos esperar del que vol que us desespereu! Algú no penssa res perdre, encara que no atenyga

340 lo que en algun temps possehir no espera. Si aquesta nimfa se mostràs enujar del que mes paraules rahonaven, assajara yo, ab enamorats serveys, mudar la sua malvolença. Però ella.s mostrava en estrem alegra de contemplar la mia bellea e no consentia les mies mans les sues vestidures toquassen.

O enujosa e cruel aygua, més fort que los murs de Troya que bastàs lunyar-

345 me de tanta glòria! Ara serà Equo venge e totes les nimfes de la mia mort se alegraran, puix ab gran supèrbia he menyspreat la humilitat de ses pregàries. Tu, més bella que totes, te penediràs que, en tan gentil edat, per causa tua, me deixe de viure. E lo món ja.s entrenyora que de la bellea de Narçiso sia orfe.

350 En tals paraules passí tan gran part del dia, que.ls cavalls de Febo dellà les colones de Hèrcules, ab cuytats passos calçigant, lo luminós carro a la posada humida del gran Occeano endreçaven. Mas, puix viu ma esperança del tot fallida, no·m poguí retraure que les amargues làgrimes, corrent dels meus plorosos uls, en la clara font no cayguessen. E del meu plor la bella nimfa tant se

355 enujava que-l seu gentil gest feÿa mostra de mi-s volgués partir. Mas, com viu que ma dolor la tribulava, ab sobresgran treball dins mi retenia la moltitut de tantes làgremes. E ab fengida alegria celava la granea de ma estrema pena, no sofrint poch afany de tenir tan gran dolor cuberta, que los plors e sospirs als qui en estrem estan tribulats gran descans porten.

36o Mas, a la fi, les tenebres de la cruel escura nit me tolgueren la vista de tan estranya bellea e los arbres, ab moltitut de verts rames, defenien la gentil font de la freda lum de Diana. Aumentà tant ma dolor, que no poguí esperar la venguda del gran Apol·lo. Ans començí tant fort a plànyer e plorar, que del carcre de tant (58r) tribulat cos volgué fogir la mia ànima; perquè, devallant

365 en los inferns, en la companyia de vosaltres de mos mals pugua millor plànyer”.

\section{DE PÍRAMUS Y TISBE}

Envides tals paraules Narçiso acabava, quant respòs Tisbe:

"La major dolor que als mesquins atribula és si en algun temps són estats

370 benaventurats. Per ço la mia pena sens comparaçió les de vosaltres sobrepuja; car, si yo he ben entés vostres querelles, jamés amor ha mostrat lur poder en vosaltres ab pròspera fortuna; ne vostres mals tenen rahó de ésser creguts, sinó sols per lo testimoni de vostres paraules. Mas, los meus, sens yo parlar, clarament se manifesten, car està lo meu cor, ensemps ab

344. enujosa: enveyosa O. Miquel i Planas (181): enu[e]josa; gli altri editori (Carbonell, 125; Martos, 190; Escartí, 107): envejosa.

344. de Troya: de la primera Troya O, lezione per la quale optano Martos (190; 380, n. 393-94) e Escartí (107).

374. cor: è la lezione di V conservata anche da Miquel i Planas (182; Carbonell, 126); 
375 l'ànima, travessat e les mies vestidures tinctes de la enamorada sanch del meu Píramus. Les vostres nafres són invisibles, e les mies porten ferea de ésser mirades. Los vostres mals demanen piadosos reçitadors, perquè als qui.ls hohiran moguen a misericòrdia; los meus, ells se manifesten e no conssenten ésser hoïts, sinó ab ulls de piadoses làgremes. Yo·m voldria

380 escusar de contar ma desaventura, mas, perquè vegau com cascú en estimar los mals propis se engana, acceptaré, entre tants altres, aquest treball e diré, ab veu plorosa, lo principi e fi de ma enamorada vida.

En un mateix jorn, exint del carçre de nostres mares, vinguem en la lum de aquest món, Píramus e yo, en aquella çiutat, los murs de la qual edificà la

385 mare de [...]no. E sola (58v) una paret defenia que la casa de nostres pares fos una. D'on se pogué esdevenir que, en aquella pocha edat que.ls chichs infants atenyen la primera conexença, coneguí yo a Píramus e fuy per ell coneguda. E no sé qual en nosaltres fon primer: amor ho conexença, tostemps seguint aquella estrema benvolença que nostra infançonia comportava.

390 Axí, aumentant lo ús de rahó en nosaltres, feÿa’s molt gran nostra benvolença; fins tant que atenyguem aquell temps en lo qual poguérem executar lo delitós servir de Venus, si no y contrastàs la crueldat de nostres pares. Emperò, assajam si en líçit matrimoni se poguera fer que, puix nostres ànimes eren juntes, los cossos separats no penassen.

395 Yo no sé què·ns defenia de tanta glòria, car, si en los matrimonis se demana egualtat, en nosaltres se trobava axí de bellea com en edat e, encara, en los béns que la fortuna atorga. Píramus no era mon pare, com Sínaras de Mirra; ni era la encantada nimfa de Narçiso. Yo tinch rahó de querellar-me de ma desaventura, car aquells qui follament e sens discreçió donen les veles als

400 vents no.s deuen maravellar si la proa de sos desigs bat los esculls, car a tart de foll navegar se ateny segur passatge. Sols la indiscreta vellea, fallida de memòria, de nostres pares lunyava nostres voluntats concordes.

Mas la estrema amor, a la qual res no li·s encobre, nos manifestà una

mentre Martos (191) accoglie cors di $O$ (e così Escartí: cos, 107), precisando che: “Tisbe [...] diu que les seues proves es poden comprovar [...] Tisbe, al final del mite, embruta el seu cos amb la sang de Píram i el travessa amb una espasa" (381, n. 428). A fortiori, non vi è motivo di respingere la lezione di $V$, dal momento che cor può stare metaforicamente per "petto"; infatti, la Tisbe ovidiana si lascia cadere sulla spada di Piramo, dopo essersene assestata la punta sotto il petto: "Dixit et aptato pectus mucrone sub imum / incubuit ferro ..." (Barchiesi ed. 2007: Met. IV, vv. 162-63.

383. de nostres mares: Martos (191; Escartí, 108) opta per la lezione del ventre de nostres mares di $O$.

384. murs: Martos (г9г; Escartí, Io8) opta per la lezione forts murs di $O$.

385. [...]no: Juno recano $V$ e $O$; viceversa il facsimile (sic) del ms. Maians di Almiñana Vallés (1983) adduce Lano. La lezione concorde di Ve $O$, Juno (conservata dai precedenti editori), potrebbe rimontare a una corruttela di Nino. Per il nome (Nino, Ninio, o Ninian, o Ninyas) del figlio di Nino e di Semiramide, costruttrice delle leggendarie mura di Babilonia, mi limito alle due seguenti testimonianze, di differente epoca: De Civitate Dei, XVIII.2: "Filium porro Nini et Samiramidis, qui matri successit in regno, quidam etiam ipsum Ninum, quidam uero deriuato a patre uocabulo Ninyan uocant” (Dombart-Kalb ed. 1955: II, 595). Quindi, Boccaccio, Teseida: "Questa Semiramìs fu moglie di Nino, re degli Assirii, et, morto il marito, veggendosi di lui uno solo figliuolo similemente chiamato Nino et questo essere fanciullo ..." (AgostinelliColeman ed. 2015: VII, 50, 208). 
secreta fenella en aquella paret que nostres cases departia; la qual, fins en

405 aquell temps, no era estada vista, car moltes coses jahen encubertes per minva de negligents descobridors. No sé a qual de nosaltres ella fon primer descuberta, que, mirant yo per la dita fenella si la bella cara de Píramus poguera veure, ja ell de l'altra part ab semblant prepòsit esperava. E, sospirant, deÿa tals paraules:

4IO 'O envejosa e cruel paret! Per què·m defens de tanta glòria, que no conssents la mia boca se acoste a la de Tisbe? Com tens poder de separar dos amants, los quals infinida amor acosta? Però, no pensses que desconega la molta gràcia que Tisbe e yo rebem de tu que dónes segur passatge a nostres enamorades paraules'.

4 I5 Tals e semblants rahons deÿa Píramus, ab veu per amor tan delicada, que no tinch poder en semblant estil aquelles reçitar.

Axí portàvem nostra amor encuberta, ab aquelles majors cauteles que amor nos consentia. (59r) E, si alguna hora la sotliçitut de nostres pares nos donava espay, sens tarda, al sobredit loch veníem. E, de continu, teníem contesa que

420 cascú de nosaltres deÿa la sua dolor ésser més estrema. Yo no curava de les acostumades cauteles de les altres dones, les quals sovint, ab fengides paraules, tenen lur amor encuberta; ans tot mon estudi era que Píramus conegués la part que en mi tenia.

$\mathrm{E}$ ab aquests treballs passàvem nostra vida enamorada, fins que un jorn, 425 venguts abduys al sobredit loch, planyent ab moltes làgremes nostra gran desaventura, diguí yo a Píramus:

'Si plànyer e plorar nostres mals reparava, ho si nostres béns, planyent, reçussitaven com los fills de la leona, seria rahó en tal treball lo temps de nostra vida despenguéssem. Mas yo no sé en què aquest tan gran dol nos

430 aprofite, sinó en aument d'estrema pena; e no podem atényer major dolor de la que tenim. Assagem la fortuna la qual, sovent, als qui la sperimenten ve pròspera.

Yo he penssat, si a tu és plaent, que, venint la nit, la qual ab ses escures ales tots los engans cobre, nosaltres enganem la sotliçitut de nostres pares, fogint ab avisada cautela en aquell temps que lo plaent dormir, alleujant los treballs, dóna repòs a totes les creatures. E siam çerts d'esperar la hu al altre, en aquella bella font, la qual fora los murs de la çiutat està, cuberta ab les verts rames de un gentil morer. E, si tu primer a la font venies, no $m$ esperes ab duptosos penssaments, car la tua Tisbe no tardara, si la mort no lo y tolia'

440 Acabant yo tals paraules, un poch espay tardà Píramus en tornar-me tal resposta:

'Entre tots mos mals, lo que més me atribula és perquè no tinch poder de acabadament mostrar-te la infinida amor a la qual justament lo merèxer teu me obligua; e per ço, guarde algun temps en lo qual sens temor pugua

420. curava de les: curave de seguir les, lezione di $O$, accolta da Martos (193; Escartí, 109).

430-431. pena; e no podem atényer major dolor de la que tenim. Assagem: viceversa, si rifâ ad $O$ (pena e pus quescu estima que maior de la que tenim no podem atenyer aseyem) la ricostruzione di Miquel i Planas, accolta anche dagli altri editori (Carbonell, 128; Martos, 194; Escartí, 109): pena. E, puix cascu estima no podem atenyer major dolor de la que tenim, assagem (185). 
445 mostrar als déus e a les gents que tinch en poch ma vida, si per tu l'avia a despendre. E per ço, no·t maravelles si vinch forçat en fer lo que tu dius, car la estrema amor que dins mi reposa me fa molt duptar los esdevenidors perills, e los pochs inconvenients me paren grans, si en dan de la tua persona (59v) s'esguarden.

$45^{\circ}$ Yo tem los mals que a tu porien seguir, perquè só çert la mort sens dupte me procurarien, la qual me seria fort enujosa sols per lunyar-me de la tua bella vista. Com serà segura cosa que una donzella en la escura nit vaja fora los murs de la çiutat? E, si los déus fins a la font te guien, com seràs allí, qui·t farà segura de les inplacables bèsties, a les quals resistir no porà la tua delicada flaquea? Per què vols fer aguayts a nostra vida?

Molts altres incovenients veig, los quals poden empachar la fi de nostre prepòsit. No·ls vull recitar perquè no cregues que·m recort d'ells per fogir al que tu tens per bo: seré alegre del que tu has penssat façam. Manant-m’o, m'o digues, perquè si qualsevol desastre m'esdevenia, tendré conort que, per

460 obeyr als teus manaments, sia en tal treball, que tot mon delit obeynt a tu alcançe'.

Envides tals paraules Píramus acabava, quant yo responguí:

'No pensses tu que yo ignore los perills que esdevenir nos poden, si seguim lo que yo desige. Mas deuries penssar que los qui tots los enconvenients

465 temen, a tart atenyen gran benaventura, e de flaquea de ànimo ve penssar tots los perills. Si tens tanta amor com tes paraules fan mostra, cobra ànimo e sies no poch alegre en seguir lo que yo vull'.

'Seguiré - respòs Píramus - pregant aquell déu qui en forma de simple toro enganà la gentil filla de Agènor sia en guarda de nostre viatge. A tu, Tisbe,

470 deman de gràçia que, si a mi seguien treballs que la mort me procurassen, no·t enuges de viure, car gran dan seria al món la pèrdua de ta vida. E més te prech no.t cuytes venir primera a la font. Espera yo y sia, perquè primer als dans ofira ma persona'.

$\mathrm{Ab}$ tal conçert e moltes làgremes nos partim, besant la paret, puix d'aldre 475 no teníem liçènçia.

Partint-me, donchs, del meu Píramus, sovint alçava los ulls a Febo. E paria’m los seus cavalls ésser tant canssats que tenien lo dia en major espay del acostumat; ho paria'm que Febo, per contemplar la bellea de Leuco, detardava son viatge, e ab la sua luminosa cara defenia la terra de les

480 tenebres de la escura nit, la qual vingué molt pus tart del que (6or) solia.

E, puix viu dispost temps per a ma partida, cobrint-me lo cap e la cara ab un vel perquè no fos coneguda, ixquí del carçre de la trista casa, acompanyantme ànimo de infinida amor. $\mathrm{E}$, si en lo camí algun murmur sentia, reclamava Píramus, car de altre déu socós no esperava. E sens dupte, tant per temor

485 com per treball, yo·m canssava; mas la sperança que de veure Píramus tenia me portava novella força. Tant que vinguí en poch espay a la bella font, a la qual Píramus encara no era aribat.

Larga cosa seria recontar los duptosos penssaments que en aquella hora ma enamorada penssa combatien.

490 Mas no estiguí molt espay que viu, per la freda lum de Diana, venir una leona, la qual paria tinta de sanch, qui, ab suaus passos, a la clara font se 
endreçava. E, per fogir a la ferea de tan brava bèstia, ab gran cuyta, lexí prop la font aquell vel ab lo qual era venguda cuberta, amagant-me en la escura ombra de una cova que poch espay de la font estava; fins que penssí que ja la

495 leona seria partida. E temia molt que, venint Píramus, se tingués per enujat de la mia tarda.

Exint, donchs, de la cova, sens que la temor no havia del tot perduda, deÿa en mi:

'O! Com contaré a Píramus los perills en què·m só vista? E serà’m pres en 500 comte de infinida amor. E, si no·m creu, mostrar-li-é los passos de la leona’.

Ab tals penssaments venguí a-quell morer, les rames del qual ab gentil ombra la clara font cobrien. E paregué'm no ésser aquell lo loch d'on m’era partida, car viu lo vert morer ab lo fruyt negre, portant dol de molta tristor, lo qual, ans que partís, ab càndida blancor relluhia.

505 Estant en aquest dupte, sentí gran remor prop la font, que penssí tornàs altra vegada la fera bèstia. E, baixant los ulls per veure si axí era, viu los cos de Píramus, qui, ab la pena de la mort, batia la sangonosa terra.

Yo no sé ab quin estil, ni ab quals paraules, poré reçitar la granea de tanta dolor. Perdí tots mos sentiments e, lançant-me davant lo fret cos, mesclades

$5^{\text {IO }}$ ab la sua sanch les mies amargues làgremes, ab plorosa veu deỹa:

'Píramus! Si la mort te dóna liçènçia, alça los ulls e veuràs la tua Tisbe'.

Ab gran pena obeý a les mies paraules, levant un (60v) poch los ulls al nom de Tisbe; los quals prest baixà, car la mort cruel havia robat les forces a la sua gentil persona.

5I5 Ab tant adolorit plant passí tan gran part de la nit, que ja Febo ab sos daurats cabells començava a il-luminar la terra. Eviu en la mà de Píramus aquell vel lo qual yo havia leixat fogint a la leona; d'on coneguí la causa de la sua mort, car estava lo vel tinct e tot esquinçat de les dents de la brava bèstia. E aumentà més ma dolor, puix coneguí la granea de sa benvolença. E rompent

$5^{20}$ les mies vestidures, ab dolorosa veu deÿa:

'Qui és aquell, per sobressavi que sia, pugua fogir a la crueldat dels inplacables fats, los quals jamés, per plors ni sospirs, muden alguna de aquelles coses que de prinçipi tenen ordenades?

O Píramus! Hon era lo teu esforç? No sabs tu que algú no·s procura la mort,

$5^{25}$ sinó per flaquea de ànimo? $\mathrm{O}$, Piramus! E per què un poch espay no perdonaves a la tua vida, perquè vesses Tisbe delliure de tots perills? Qui·s deu alegrar, encara que de un gran perill sia estalvi? O, cruels déus! Per què·m delliuràveu de la leona, puix sabíeu que la mort ab tan gran pena me era vehina? Mas, què aprofita lo plànyer sobre cosa irreparable, car sols la mort

530 és sens repar? Però, puix ab moltitut de làgrimes no puch reçussitar la tua vida, plau-me lo morir, que la vida sens tu en estrem m'és enujosa. Mas, perquè la causa de nostra mort per a sempre sia palesa, prech als déus aquest

501. a.quell: Miquel i Planas (I88) e Carbonell (130) optano per la lezione fins en aquell di $O$; en aquell Martos (i97; Escartí, III).

507. mort: cruel mort O, lezione accolta da Martos (i97; Escartí, III).

518. tinct e tot: tinct de sanch e tot legge Miquel i Planas (I89), seguito dagli altri editori (Carbonell, I3O; Martos, I98; Escartí, III), sulla scorta di $O$ (tint de sanch tot). 
arbre jamés no consenten que lo seu fruyt leixe lo dol de nostra desaventura. E als déus més soplich, puix en vida a nostres desigs tant han contrastat, ara, 535 aprés mort, ordenen que.ls nostres cossos en un mateix sepulcre reposen’.

Encara dient tals paraules, me lançí damunt aquella espasa que del cos de Píramus sangonosa exia, leixant ma vida entre sos frets braços. Han-me portat los fats en aquest trist verger, en lo qual sempre les desiguals penes de amor nos tribulen".

\section{Apparato}

1 Lamentaçió... Çínaras] Lamentacions de Mirra e Narcisso e Tisbe (dopo Tisbe: un tratto dicancellatura) per mossen corella (corella in interlinea) cavataller e en sacra Theolagia professor $O-2 \mathrm{Ab}$ desig: desig preceduto dallo spazio in bianco riservato all iniziale e ab scritto sul margine sinistro $O$; a] en $O-3$ e] om. $O-3-4$ que al ... Sibil.la] quel desterrat de troya mostra la carrera a Sibilla $O-4$ al] als con s depennata $V$; dolorit $O-5$ en lo qual] hon $O$; en continu plor] om. $O-6-7$ a Mirra ... treballant] Mirra e Tisba ensemps ab Narciso appartats dels altres de sos mals en fort manera planyent e treballant $O-8$ Però ... començà] pero comensa primer Mirra $O$; reçitar] dir $O-9$ dopo desaventura uno spazio in bianco al centro del quale si trova, con modulo di scrittura maggiore, come titolo, Mirra, preceduto da paraffa $O-10$ condició] inclinacio $O-12$ ab vel] sots vel $O$; nostre voler la desiga] lo tal voler nostre demana $O-12-13$ a mi] om. $O-13$ e digna de gran mèrit] om. $O$; dopo amar] vi depennato $V$; amar a $O-14$ de prínçeps e reys] de grans reys $O-15$ partir-me] apartar me $O-17$ puix] pus ja $O$; dopo promesa] e la tua bellesa contrasta als frets vots de diana $O$; digues-me] digues $O-18$ contenta de $O-19$ per la mia cara de vergonya colorada cahien] per vergonya per la cara acolorada me cahien $O-20-21$ ploràs per temor de] temes de $O-21$ perillosa] tamarosa $O$; dopo la qual] sovint $O-22-23$ e, lohant la mia vergonya, ab les sues mans sechava les abundants fonts de mes làgremes] levant me la mia casta vergonya ab les mies belles mans cessaven les abundants fonts de mes lagrimes $O-23$ les sues abundants $V-24$ lo primer aradre] la primera errada $O-25$ possehir] levar $O-26$ desige] vull $O$; en res a vós] a vos en res $O-27$ a aquell] en aquell $O-28$ salut] sanitat $O ; \mathrm{E}$ om. $O$; a aquella] a la $O-29$ fallença per no poder dir] falensa per a $\operatorname{dir} O-30$ ànimo] nom $O-31$ desordenat] deseventurat $O$; no podent resistir] nos pot retenir $O$; en mi deÿa] deya en mi $O-32$ e hon] e no $V$ - 33 culple $O$; en] ab $O-34$ obliden qualsevol] oblidant se de qual se vol $O-35$ per] a $O-36$ de aquell qui l’à] de qui es $O-37$ celçitut humana, enemigua y envejosa del meu delit] solicitut humana enveyosa del meu sobiran delit $O-38$ defenen] ab grans penes deffenen $O-39-40$ en una part del món ... vergonya] om. $O-40$ dopo no] ho sembra depennato $V-41$ lo meu desig] lo desig meu $O-42$ ni en alguna part poden ésser bones] ni alguna part james son bones $O-43$ podien $O-46 \mathrm{erra}$ om. $O$; E, perdent seguito da e perdent depennato $O-47$ dopo mort] de mon pare depennato $O$; desesperada] desemparada $O-48$ content] alegre $O-49$ que jo repose entre los seus bells braços] de reposar entrels seus brassos $O-50 \mathrm{E}] \mathrm{om}$. $O$; ne reporte] re (depennato) ne reposte $V-50-51$ no sens gran treball se deixen] no ab poch carrech se leixen $O-51$ fon Júpiter] Jupiter fou $O$; patricidi $O-52$ dopo paren una lettera depennata $V$; lo] om. $O-54$ contrasts] combats $O$; combatien] om. $V$ - 55 e, amonestant] amonestava $O-56$ atorgà repòs als] atorgue repos e als $O$; pensses] penes $O-57$ callada] calda $V-58$ enamorades pensses ab més poder] persones ab doble poder $O-59$ aumentava; e, quasi] tant augmentava que quasi $O-60$ dopo son] me depennato $V$; trista] om. $O-61$ la companya $O-62-63$ per fexugua ... enugs] peresat per fexuosa carreguada (carreguada con da depennato) de deseguals anuigs sobrel meu lit $O-63$ enug $V-63-64$

532-533. prech als déus aquest arbre...lo seu fruyt leixe: per la corrispondente lezione di $O$, prech en aquest arbre que james consente quel seu fruyt leix, vid. Intr., n. 6.

539. A seguire, dopo tribulen, il Jardinet de orats riporta un frammento del prologo della Glòria d'amor di Rocabertí (vid. Apparato). Lo stesso frammento figura nel Cançoner del Marquès de Barberà (ms. 992, Biblioteca del Monestir de Montserrat, f. 138r.) e in un codice petrarchesco della Biblioteca di Ramon d'Alòs Moner (ms. 824.4 N=49.9, f. 11r). Per il primo, vid. Martí (1997: 477); per il secondo, Villar (1995: 62-64). Il frammento è oggi considerato pressoché unanimemente "lligat accidentalment a Roís de Corella" (Bassegoda Pineda, 2011: 160): Cingolani (1997: 71-72), Martos (2001a: 99-102), Martos (2002: 60), Pellisa (2013: 112). 
no sabia qual millor que mort elegís. E] no sabia qual part millor me elegis (me elegis depennato) que mort elegis mes $O-65-66$ e fóra millor assajar en vida ... portaria] foram millor assegurar me en vida si tal infamia me aportas $O-68$ qui $O-69-70$ ab tant desordenat voler] ab tant gran desorda $O-70$ tampoch] om. $O-71$ yo elegis $O$ - 72 sempre] per a sempre $O$; remeyaven $O$; l'ànima del cos] la mia anima del noble cors $O$; passions] potencies $O$ - 73 infançonia ] inflamada (con da aggiunto in interlinea) infançonia $O-74$ a quelsevol $O$; recorren $O-75 \mathrm{ja}$ ] om. $O$; quasi preceduto da qus depennato $V-77$ ignoçènçia] ignorancia $O$ - 79-81 vellea, freda ... restaurar ma vida] vella freda inimiga de ma casta ignorancia que no consentint dormis ferma aquella nit la qual sola podia restaurar ma vida $O-82$ mes darreres paraules ... venia] mes paraules que manifestaven la dolor de aquella don venien $O-83$ de acórrer] en acorrer $O$; aportave $O-84$ tremoloses mans] temoroses paraules e mans $O$; aquell las] aquelles $O-86$ vegades] voltes $O-86-87$ puix que.l cos hagué cobrat sos] pus viu que lo meu cors recobrats havia tots sos $O-87$ veu tremolant] tremolant veu $O-89$ la leona $O-91$ és de elegir] has elegit $O$; si] pus $O-93$ yo sospite] yo sospitosa $O-94$ ésser-ne] esser $O$; recorda $O-95$ se matà, que si voluntàriament hagués] se mata voluntariament que si hagues $O-96 \mathrm{E}$ més si era ignoçenta ... justes leys] e mes era de empatxar les justes leys les quals $O-98$ si són tinctes de sanch ignoçent. Mas] om. $O$ - 98-99 lo remey ... tens] lo remey de la mort est certa no pot fallir $O-99$ per (in interlinea $V$ ) algun espedient (espedient preceduto da lo depennato $V$ ) ab algu altre spedient $O-101$ dir-me la dolor ... atribula] dir qual es la dolor que la tua anima atribula $O-102$ al que] al qui $O$; acabat] de mi acabat $O-103$ atribulats $O$; passió] doler $O-105$ pereosa] pererosa depennato e seguito da pahorosa $O$; gran] om. $O-106 \mathrm{E}$ ] om. $O$; te ha] te te $O$; poder absolut] absolut poder $O-107$ al qui.t parrà ésser culpable] lo quit sera culpable $O-108$ leixes $O$; les virtuts $O-109$ amagadament] om. $O$; venjança] venge $O-$ $109-110 \mathrm{E}$ si pensses contra tu los déus sien yrats] pençes que los deus contra tu sien yrats $O-110$ tenyierem ... sacrifiçis] tenyirien lurs archs de honrat e pleccats sacrifficis $O-111$ amanssar] de amençar $O$; la lur] la sua $O$; lur] llur propri $O-112-113$ per pèrdua ... matant-se] de perdua o alguna persona (persona seguito da a alguna persona depennato) aqui en strem ames recort te quant mal repara Dido car metant se sos mals comensaren $O-113-115$ No pensses en los mals abssents ... dels esdevenidors] no penses ab los absents sino ab les coses pessades de les quals hom se deu servir sol per exemple de les sdevenidores $O-116-117$ la qual, si vols, te farà (fare $V$ ) possehidora de no poch gentil enamorat] e si vols te fara poseir no poch gentil enamorat $O-119$ e elegeix] elegeix $O$; plasent] plaer $O$; desig] delit $O-120$ pocas arades $O$; sofiriariria $V$; nova] om. $O-120-121$ los lauradors] tots los leuradors $O-122 \mathrm{car}$ ] om. $O-123$ honrat] honrat e $V$, leggibile fino a honra in O per rifilatura del margine successiva alla copia del testo - 124 mot de 'pare'] nom pare $O$; plor] por $O$; la paraula] om. $O-125$ que penssà] e pensa $O$; essent yo] yo essent $O-126$ castich e] castich o $O$; Ab] e ab $O$; les mans fredes] les sues fredes mans $O$ - 127 humida cara] care humida $O$; demanà'm altra vegada] altre vegada demana $O-128$ prometent-me] promatent $O$; paraules] juraments $O-129$ sabera $O-130 \mathrm{E}]$ om. $O$; los meus] mos $O-131$ jurà] per los deus me jurave $O-132$ Yo] O yo $O-133$ sospita $O$; a tart] $\operatorname{tard} O$; Però] mas $O-133-134$ per dar loch a la] om. $O-134$ tan animosa vella] tant anuiosa velesa $O$; tra sobres e gran una p depennata $O-140$ ab honesta fama, que, vivint, morir de eterna infâmia] en eterna memoria $O-141$ qui] que $O$; leys humanes] humanes leys $O-142$ a qui] qui $O$; ab molt estret grau] en extrem grau $O-143$ saber ab gran pressa] ab gran pressa saber $O-143-144$ persona al món benaventurada sia no estime] no crech persona al mon beneventurada sia $O-144$ sola] sols $O-145$ dopo vergonya] la qual sovint de extrema amor es inimiga $O$; doloroses $O-148$ poguera] pogues $O$; véu] viu $O-149$ 150 si poria restaurar] restaurar $O$, con la seconda r in interlinea - 151 ni.t] not $O-152$ e hagué $O-153$ alargat] elegrat $O$; eren sotlíçits] e esser sollicits $O-154$ desonrada] desordonada $O$; de mi pendrien venjança] en mi prenguessen venge $O$; E] om. $O-155$ festa de] feste a $O$; [..] es] ço es $V$, om. $O-156$ matrones] nacions $O$; corones $O-157$ ares preceduto da arres depennato $O$; per fer] per affer $O-158$ apartant-se de la religió de Venus, leixaven per nou dies] apartaven se de lur religio de venus e leixaven $O$; leixaven] om. $V$; sos] lurs $O-159$ temps in interlinea $O-160$ dient] dient li $O-161$ de qui] de que $O-162$ car] que $O$; tual tua filla $O-163 \mathrm{ja}$ om. $O-164$ leixar $O$; la calor] la color $O-165$ general] egual $O$; acceptad $O-166$ qui] que $O$; ab] om. $O-167$ hora] seguito da Sinarras depennato $O$; ab gran desig, Sínaras] en gran desig Sinarras $O$; tant] preceduto da nit depennato $O-170$ pecat] cors $O-170$-171 Mas la certa esperança ... erra esperàs] mes certa speransa que del sdevenidor delit tenia me feya oblidar la granesa de tant crim e qualsevulla pena de tant gran pena (pena depennato) erra speras $O-173$ dopo Pres-me] donchs $O$; per la mà la esforçada vella, la qual] la sforsada vella per la ma (ma in interlinea) qual $O-174 \mathrm{E}$, entrant] entrant $O-176 \mathrm{Aparta}$ a apagant $O$; aporta $O-179$ portaren esforç de entrar en la escura cambra] aportaven força de star en la tenebrosa cambre $O$; tenyint de verge] tenyint la verge $O-180$ los quals] les quals $O-181$ sovent con un segno dinasale in interlinea semiraschiato $V$; esguardant ma poca edat, me deÿa] sgordant me deya $O$; e] om. $O$ - 182 apartava] treballave (in interlinea) apartar (con r finale scritta su un precedente apartaven $O$; tota] tata $O-183 \tan$ om. $O-184$ aportas $O$; quasi] om. $O-185$ voluntat] 
la voluntat $O$; me] yom $O$; dins] en $O-185-186$ un fill e germà, nét e fill] un fill e net $O-187$ que per tant poch] ab tant poch $O-188$ de mon peccat] de tant gran peccat $O-188-189$ desigà Sínaras saber] desijave saber Sinaras $O-189$ que] qui $O$; semblava a Mirra] semblave Mirra $O-190$ dels enamorats engans] dengans $O-191 \mathrm{E}] \mathrm{om}$. $O$; gran] om. $O$; paraules] les paraules $O$; per arrependrem $O-192-193$ ab cuytats passos, corregué a la espasa que prop si tenia, e asajà] recorrent a la spasa que prop si tenia ab cuytats passos assaga $O-193$ piadosos déus] deus piedosos $O$; esforç] forsa $O-194$ ensutziar] ensutzar se $O$; en pròpia] en la propria $O-195 \operatorname{del}$ tot] om. $O$; conexenta con a depennato $O-196$ los déus] als deus $O$; Mas] om. $O-197-198$ no plagué als déus] no consentiren $O-198$ fins que] fins tant $O-199$ la un] lo $O$; rahó] de reho $O-200$ E, perquè] pero perque $O$; no] om. $O-201$ major] mes $O-202$ legea de mon peccat] justa penitencia de mon peccat $O-203$ lo qual] la qual $O$; làgrimes] mies amargues lagrimes $O$; amargua] desaventurada $O-205 \mathrm{De}$ Narçiso] om. $O-206$ No] Yo no $O$; parlara $O$; deixà] leixara $O-207$ alsant $O-208$ endressant $O$; de Biblis] debils $O-209$ doloros $O-210$ semblant] tal $O$; dopo plànyer] uno spazio in bianco al centro del quale si trova, con un modulo di scrittura maggiore, come titolo, Narcisso, preceduto da paraffa $O$ - 212 tribulen $O$; major descans] delit $O-213$ mals] tra 1 e s una lettera depennata $V$ - 214 Digua] e diga $O-215-216$ justament contrastant al desorde de amor desonesta] om. $O-221-222$ la apartada religió de Diana] la partida e la religiosa diana $O-223$ la caça] cassa $O-223-224$ que la vida humana porta] que ab si la vida humana aportava $O-224$ per contemplar a mi] de contemplar en mi $V$; leixaven $O-225$ que] qui $O-227$ més que] mes de $O$; de mi.s] de mi (scritto in interlinea) se $O-228$ de] om. $V$; les darreres] de les derreres $O-229$ parlar] poder $O-229-231$ perquè tenia per costum ... amagats plaers de Júpiter] perque mentre que Jupiter ab les Nimphes ab gran delit reposave tenia per costuma Echo ab gentils paraules detenir la gran deessa que fugint les Nimphes eran cuberts de Juno los furtats plers de Jupiter $O-232$ poderosa] tant poderosa $O-232-233$ de Júpiter los adulteris] los enguans de Jupiter $O-234$ havien fi] feya $O$; volgué que] volgue $O-235$ tanta fallença que] falensa e que $O$; lo darrer so] lo so derrer $O-237$ la nimfa] Nimpha $O-238$ dir corretto su dia $V-239 \mathrm{Y}]$ om. $O-239-240$ ella son costum, digués ... de mi hohia] lo costum seu aqueixes mateixes paraules que de mi hoy $O-241$ de continu] de continent $O-241-242$ amor de Venus] venus $O-242 \mathrm{mes}]$ les mies $O$; endrecava $V$; secondo als] om. $O-242-243$ en lo servey de la casta deessa] e en lo honest servey de la casta Diana $O-245$ parlaria] diguera $O$; ella] ell $O-245-246$ se pogués la sua dolor fer palesa] pogues fer la sua dolor palesa $O-246-247$ estrema bellea] bellesa extrema $O-247$ Tant que] tro atant que $O-247-248$ enujosa calor] extrema calor anuyosa $O-248$ y] om. $O$; dopo vert] ab depennato $V-250$ gran Febo] sobiran apollo $O$; totes les] les $O-251$ no] nom $O-252$ formà tals paraules] acabadament forma $O-253$ que] car $O$; tenia] havia $O-254$ paraules] rehons $O$; diguíl yo digui $O-255$ porta] me aporta $O-256-258$ Mas la enamorada ... pena porta] om. $O-259$ en mi loch] loch en mi $O-259-265$ que yo esperava ... Per què fuigs de mi] car si yo sperave si aquell suau vent Zephiron vendria e vahent la sua tarda digui suau Zephiron pus per costum tens sovint de gran calor deffensar fes me desliure ara per que fuig de mi a les quals paraules respos Echo per que fuig de mi car de mes dir no tenia licencia $O-266$ que] qui $O-267$ Ans asajà, venint ... meu coll] asseia de venir devers mi lansant los seus brassos bells sobre lo coll $O-269$ dopo vixcha] mas pus veu de son voler lesperansa del tot perduda depennato $O-270$ fort] $\mathrm{om}$. $O-273$ de la sua dolorosa] de sa $O-274$ Tant, que amagrí la sua gentil persona] fins que amagri tant la gentil persona $O-275$ soferí] li sofferi $O$; en dures] ab dures $O-276$ aquella preceduto da a depennato $V-277$ passant $O$; descuydada] desdenyada $O-278$ qui] que $O-279$ veÿa] vehee $O$ - 279-281 la fortuna, que de continu aguayta als ... adverssa] la fortuna qui de continuus aguayts com vers mi se mostraria adversa $O-282$ stimant $O$; ni] o $O$; ella] om. $O-284$ als] los $O-285-286$ a la suau armonia de concordants veus] ab la melodiosa (melodiosa scritto in interlinea su suau depennato) ermonia de suau e gentils veus concordans $O-287$ les condicions $O$; qui] que $O-288 \mathrm{ab}$ ] en $O$; e]o $O-289$ contenta. Fins tant que, un dia, enujat] tro atant contenta que hun jorn anujat $O-290$ lo servir] la servitut $O-291$ axí] om. $O$; encircuien $O$; no comportaven] contrestaven $O$; se calfâs] sescalfas $O-292$ grans] om. $O-293-294$ e bella] om. $O-294$ mig] lo mig $O-295$ de tal aygua] de tant concordant aygue $O-295-296$ a les erbes, que] als arbres qui $O-296$ prop li] prop a li con a in interlinea $O$; verts $O-297 \mathrm{E}$ fuy $O$; de acceptar $O$; deixar] lunyar $O-298$ que] qui $O$; la florida erba] en terra $O-300$ d'enteniment] de seny $O$; qui] aquell qui $O$; algun·altra lohàs] lohas algun altre $O$ - 301 maravellat per l'estrem de tanta bellea, la primera set tenguí perduda] fuy tant torbat mirant lestrem de tanta bellesa que la primera set tingui oblidade (obligade con correzione dig in d) $O-302$ tenia poder] tingui poder $O$ - 302-303 tenia atreviment banyar les mans en la freda aygua] havia atreviment de abaixar les mans en laygue $O$ - 303 la tan] tant $O$; nimfa] nimfa depennato e seguito da nimfa $O$ - 304 que més] car mes $O$; hoyr d'ella] della hoyr $O-305$ que a mi] car a mi $O-306$ en mi] ab mi $O-307$ sens larguea de temps] om. $O-307-309$ que $\cdot \mathrm{m}$ forçà yo primer, en semblant ... obligava] que yo primer digui en semblant stil aquanta amor novament la sua vista me obligave $O-310$ Quant tinch agrahir] e quant tench que grahir $O-311$ bosch] loch $O-313$ perquè.l] per 
aquell $O$ con a $e$ l finale raschiate $O$; en major] ab meior $O$ - 314 que més ] car més $O-315$ sens que] que sens que $O$ - 315-316 d'elles, ni poch amar ... guardó] de aquelles ne de pocha amor a tu en cara que non speres guardo $O$ - 316 que] car $O-317$ mereix ésser possehida] merexia esser presa $O$ - 317-318 font, car ... algú sia] om. $V-318$ asegure't la tua] asseguret en la tua $O-319$ en la qual] a la qual $O$; com a Déu de qui] com ade qui $V-320$ en plaure] en lo plaure $O-322$ no gens] e no gens $O$; que] car $O-324$ la major] maior $O$; ma] om. $\mathrm{V}$; que] car $O$ - 325 concordes] om. $O-326$ tant que] tant $O$; ses] les $O-328$ les sues paraules] que les paraules $O-329$ que, yo] car yo $O-329-330$ e ans responia ... acabassen] om. $O$; sua] om. $O-331-332$ ab la mia boca tocar la cruel] atocar ab la mia boca la tant cruel $O-332$ que] car $O-333$ gentil] tant gentil $O-333-334$ tant que ab la cara tocava la enemigua] tro atant que yo tocave la tant inimigue $O-335$ fogir] de fugir $O-337$ veure's] veureus $O$ - 338-339 aquella que us promet ... que us desespereu] aquella (con a iniziale in interlinea) qui promet sens voler de attendre e aquella quels met en speransa del que vol ques desesperen $O-339-340$ no atenyga lo que $\ldots$ no espera] no (scritto in interlinea) alcansa lo que no creu possehir $O-340$ nimfa] tant bella nimpha $O-341$ asseiarea con a finale depennata $O$; ab] scritto su als $O-343$ consentia les mies ... toquassen] consent les mies mans sol la sua vestidure toquen $O-344$ enveyosa $O$; fort preceduto da fot depennato $V$; de Troya] de la primera Troya $O-344-345$ que bastàs lunyar-me de tanta glòria] pus tu sola bastas amudar me de tanta gloria $O-346$ humilitat] mansuetut $O$; ses] lurs $O-348$ deixe] leix $O$; que de la bellea] que la bellesa $O-351-352$ ab cuytats passos ... endreçaven] calsigaven $O-352-353$ viu ma esperança del tot fallida] viu fallida del tot me speransa $O$ - 354 plorosos] om. $O$; la clara] clara $O-355$ que.l seu gentil gest] que al seu gest $O$; mostrar con r finale depennata $V$; Mas, com] pero pus $O-356$ que ma dolor la tribulava] que de la mia dolor ella tant se tribulave e $O$; reteni $O-357$ fengida alegria celava] infinida alegria callave $O-358$ no sofrint] e no sofferia $O$; tenir] retenir $O$; cuberta] en cuberta $O-358-359$ que los plors ... tribulats] car los (los preceduto da los depennato) plos e los sospirs del qui enstrem (enstrem con s inserita tra $\mathrm{n} e \mathrm{t}$ ) son atribulats $O-360$ escura] scu depennato seguito da scura $O-361$ estranya] infinida $O$; e los arbres] car los arbres $O$; ab moltitut de verts rames] ab llurs brassors $O$; la gentil preceduto da gen depennato $O-362$ Aumentà] e axi augmentave $O-363$ Ans] Mas $O-364$ de tant tribulat] del tant atribulat $O$; perquè] per ço que $O-367-368$ De Píramus y Tisbe. Envides tals paraules ... respòs Tisbe] Envides Narcisso les semblants paraules respos Tisba. Tisbe (Tisbe al centro di uno spazio in bianco, con modulo di scrittura maggiore, come titolo, preceduto da paraffa) $O-369$ atribulat con t finale depennato $O$; si en algun temps] si james $O-370$ la mia pena] lo meu dolor $O-371$ yo he ben entés vostres querelles] yo o he be entes les nostres $O-373$ Mas] pero $O-374-375$ cor ... l'ànima] cors ensemps ab lanimo $O$ - 375 vestidures leggibile fino a vestidu in $O$ per rifilatura del margine; de la] ab la $O$; del] e del $O-376$ Piramus preceduto $d a$ pri depennato $V-377$ perquè] per quel con 1 depennata $V-377-378$ als qui.ls hohiran] los quim hoyran $O-378$ a] ab con b depennata $O-379$ ulls de piadoses] ulls humils de piedoses $O-380$ recomptar $O$; vegau] veien $O$; com cascú] quant quiscu $O-381$ engane con segno diomissione di nasale depennato $O$; entre tants altres] entrels (con ls aggiunto successivamente) altres $O-381-382$ diré, ab veu plorosa, lo] dir ab plorasa veu lo $O-383$ jorn] dia $O$; carçre] carcer del ventre $O-384$ murs] forts murs $O-385$ [...]no] Juno $V$ e $O$; que la casa] ques (con s raschiata) les cases $O-386$ pogues sdevenir $O-388$ qual en nosaltres fon primer] qual primer en nosaltres fon $O-388$ 389 tostemps seguint aquella estrema benvolença que nostra infançonia comportava] car tots temps segui aquella mes extrema (extrema seguito da bellesa depennato) amistat que nostre infantesa nos conportave $O-390 \mathrm{E}$ axi $O$; lo ús de rahó en nosaltres] en nosaltres lus de reho $O$; fë̈a's] fem $O-391$ benvolenca $V-391-392$ fins tant que atenyguem ... poguérem executar] tro atant que attes aquell temps en lo qual poguem exercitar $O$ - 393 Emperò] pero $O$; poria $O-394$ coniunctes $O-395$ demanave $O-396$ ygultat $O$; de bellea] en bellesa $O$; e, encara] leggibile fino a en enca in O per rifilatura del margine - 397 la fortuna] furtunans $O-398$ prima di encantada] encando depennato $O-398-399$ ma desaventura] furtuna $O-399$ sens discreçió] sens discrecio axi com vosaltres $O$; als] a les $V-400$ marevellar preceduto da mare depennato $O$; sos desigs] lurs delits $O-401$ attenye con e finale depennata e con segno dinasale depennato $O$; Sols la indiscreta vellea] sola la indiscreta bellesa $O$ - 402 lunya $O-403$ la estrema] a la fi (fi depennato) extrema $O$; no li-s encobre] nols sta encubert $O-404$ secreta] streta $O$; paret preceduto da fenella depennato $O$; en] om. $O-405-406$ encubertes per minva de negligents descobridors] encubert per fretura de negligencia de (de in interlinea) descobridors $O-406$ a qual] per qual $O-407$ yo per la dita fenella si] yo si per la dita fenella $O-411$ Tisbe] Pyramus $O$; poder] atreviment $O$ - 411-412 dos amants, los quals] dues persones les quals $O-412$ acosta] ajusta $O$; pensses que] penses $O-413$ Tisbe] Pyramus $O-415$ rahons] paraules $O-416$ estil preceduto da poder depennato $V$; aquelles reçitar] recitar les $O-417$ amor] amistat $O$; majors] millors $O-418$ donava] dave $O-419$ tardar $O-420$ quiscu $O$; més] molt $O$; curava de les] curave de seguir les $O-422$ encuberta] ab cubert $O$; tot] om. $O-423$ en mi tenia] tenia ab mi $O$ $-424 \mathrm{E}] \mathrm{om}$. $O$; vida enamorada] enemorade vida $O-424-425$ fins que un jorn, venguts abduys al] tro atant que 
hun jorn venint en lo $O-425-426$ gran desaventura] desventura $O-427 \mathrm{Si}$ plànyer e] sil planyer o $O-428$ los fills] lo fills $O-428-429$ de nostra] de la nostre $O-429$ despedre $O$ con segno di nasale e non leggibile oltre per rifilatura del margine - 429-430 en què aquest tan gran dol nos aprofite] a que alre aquesta gran dolor aprofite $O$ -430-431 e no podem atényer major dolor de la que tenim. Assagem] e pus quescu estima que maior de la que tenim no podem atenyer aseyem $O-431$ als qui] als qui $O$ con i scritta su e -437 cuberta ab les verts] en cuberta ab les verdes $O-438 \mathrm{tu}] \mathrm{om}$. $O$; font] dita font $O-438-439 \mathrm{no} \cdot \mathrm{m}$ esperes ab duptosos penssaments] no spers ab duptosa speransa $O-439$ lo y] lay $O-440$ tals] les semblants $O-442 \mathrm{mos}]$ los $O$; més in interlinea $O$; perquè] que $O$ - 443-444 justament lo merèxer teu me obligua] lo teu merexer justament me obligue $O-444$ guarde] aguardar $O$; pugua] yo pugue $O-445$ que] om. $O-445-446$ l'avia a despendre] lavia despendre $O-446 \mathrm{E}] \mathrm{om}$. $O$; vinch] tinch $O-447$ que] qui $O$; molt] $\mathrm{om} . O-448 \mathrm{dan}$ ] sguard $O-452$ fora] fora (depennato) sola fora $O$ - 453 allí] aqui $O-453-454$ farà segura] assegurara $O-454$ inplacables bèsties] feres inpecables bisties $O$; a les quals] a la qual $O-455$ flaquea] natura $O-456$ poden] poran $O-457$ cregues] penses $O-458$ seré] e sere $O$; penssat façam] pensat que y fassa $O-458-459$ Manant-m’o, m’o digues] manant mo digues $O-459$ si qualsevol desastre m'esdevenia] qualsevulla cosa nesdevengue $O-460$ sia] sere $O-462$ tals paraules Píramus acabava] les tals tals (secondo tals depennato) rehons acabave $O$; yo] om. $O-463$ seguim] aseguim con a depennata $O-464$ $\operatorname{desig} O-465$ beneventurança $O$; e] om. $O-466$ amor] temor $O$; tes] les tues $O$; fan mostra] mostren $O-469$ Agènor] Agenor scritto in interlinea su [?]eneto (dubbia la lettura dell'iniziale) depennato $O$; sia] que sia $O-470$ que, si] qui si $O-471$ dan] om. $O-472$ venir] en venir $O$; Espera] e spera $O$; yo] que yo $O-472-473$ als dans] al demenys $O-473$ ma] la mia $O-474$ d'aldre] de mes $O-476$ alcava $V$; alsant $O-478$ ho paria'm que Febo, per contemplar la bellea de Leuco] e podie esser que per complir Apollo la bellesa de Leclonto $O-480$ del que] que $O-481$ dispost con i scritta su una e $V$; per a] per $O-481-482$ lo cap e la cara ab un vel] la care $O-482-483$ ixquí del carçre de la trista casa, acompanyant-me ànimo] isquim del carcer ab animo $O$ - 483 murmur] parlar $O$; reclamava] reclamave a $O$ - 484 altre déu socós no esperava] altres deus no tenia (no tenia depennato) secors no sperave $O-484-485$ tant per temor com per treball, yo $\cdot \mathrm{m}$ canssava] car per treball yo cansave $O-486$ novella força] novell esfors $O$; espay] om. $O-487$ encara] om. $O-488$ recontar] recitar $O-490$ no in interlinea $O-491$ paria] me paria $O$; qui] om. $O$; se] om. $O-492 \mathrm{E}$ ] e yo $O$; a la ferea de tan brava bèstia, ab gran cuyta] ab gran cuyta a la faresa de tant brave bistia $O-493$ amagant-me] e amagant me $O-494$ poch] a poch $O-494-495$ penssí que ja la leona seria] pensi la leona sen seria $O-496$ tardança $O-497$ la temor no havia del tot] del tot la temor no havia $O-499$ què·m] que $O-500$ no $\cdot \mathrm{m}]$ nou $O$; los passos] en la terre los possos $O-501$ tal pensament $O$; a.quell morer] fins en aquell arbre $O-503$ lo fruyt] fruyt $O-504$ ab càndida blancor] om. $O-505$ gran remor prop la font, que] tant gran remor prop que $O-506$ la] le $V$; abaxant $O$; axí era] era axi $O-506-507$ de Píramus] del meu Pyramus $O$ - 507 mort] cruel mort $O$ - 508 quales $O-509$ lancant $V-509-510$ mesclades ab la sua sanch ... ab plorosa veu] mesclave ab la sua sanch les mies amargoses lagrimes mes ab veu piedosa $O-511$ Pyramus Pyramus $O-512$ obeyra $O-513$ mort cruel] cruel mort $O-515 \mathrm{Ab}] \mathrm{En} O$; sos] om. $O-516$ a il.luminar] de illuminar $O$; de] de seguito da de raschiato $O$ - 518 tinct e tot] tint de sanch tot $O$; de les dents de] de les ungles cruels de $O-519$ més ma dolor, puix] tant (in interlinea) ma dolor que puys $O-520$ dolorida $O-522$ sospirs] per suspirs $O$; alguna] nengunes $O-523$ de prinçipi] del principi $O$ - 524-525 Hon era lo teu esforç ... O, Píramus] om. $O-526$ perills] treballs $O-529$ prima di Mas] O Pyramus hon era lo teu esfors, no sabies tu que nengu nos procura la mort $O$; cosa] cars $O-530 \mathrm{ab}]$ lab con 1 raschiata $O-531$ que la vida] e la vida $O-532$ 533 prech als déus aquest arbre jamés no consenten que lo seu fruyt leixe] prech en aquest arbre que james consente quel seu fruyt leix $O-533$ desventurança $O-534$ més soplich, puix en vida a nostres desigs] suplich que pus en vida en nostres delits $O-535$ mort] nostre mort $O-536$ tals] les tals $O-537$ exia] exida era $O$; sos] los seus $O$; aportat $O-538$ sempre] per asempre $O-539$ atribulen $O-A$ seguire, dopo atribulen: Vosaltres jovens en la pensa dels quals amor continuament habita los enteniments de pietat elevats hoyau de les mies lamentacions la nova gloria e secreta elegria que a la deytat de Cupido sestenen. Entrant en lo jardi de amor viu los arbres grans pero spinosos que qui fort los streny sent infinide dolor. Los fruyts de sebor dolça semblansa tenen. Mas en aquells havia tantes doloroses pacions que lo dit del rebie a la summa de les penes no bastave aquell iardi de una natural voluntat e inclinacio se concep per affectat desig que dels ulls principi ve los quals representen la delitosa cosa a la ociosa pensa e tant fort desperta la voluntat que no podent servar dret ni orde passa los termens de la reho e per la via de la pensa a la memoria recomane los dolorosos pensaments de sa natural costuma. E es de tal condicio qui en aquell vol entrar que tant quant de sos delits mes vol sentir viu ab meior dolor. Ffinis $O$. 


\section{Apparato complementare}

4 son $O-5$ recompten $O-7$ maior $O$; llur $O-10$ fugir $O$; inpossible $O-11$ subira $O$; attenyer $O$; desijam $O$; encare $O-12$ strem $O$; ho] o $O$; hombre $O$; aparague $O-13$ piedosa $O-14$ altre $O$; menyspresant $O-15$ pensave $O$; belesa $O-19$ lagrimes $O-20$ fresques $O$; pensave $O-21$ plasent $O-24$ meravella $O$; demenant me $O$; altre $O-25$ parria $O$; digna $O$; licit $O-26$ desemble $O-27$ strema $O-30$ ho] o $O-32$ speres $O$; inmortals $O-35$ aiusten $O$; eugua $O-36$ lo aucell $O-37$ concebut $O$; Mes $O$; enamigues $O-39$ oyt $O-41$ ser $O$; strem $O-42$ secondo ni] ne $O-43$ ésser] ser $O$; perjuy $O-44$ triunpha $O-45$ desempara $O$; propria $O-46$ desliber $O-47$ attenyare $O$; ho] o $O$; venyadora $O$; Mes $O-48$ contrastem $O$; tolre $O$; Sinarres $O-49$ piedosament $O$; no se anuya $O-50$ pense $O$; stima $O-51$ attenyer $O-52$ senyoraiar $O$; cel $O$; maiors $O$; perquè.ls] perque los $O$ - 53 triunpha $O$; legesa $O-54$ mentre $O$; pensa $O-55$ plasent $O-57$ pensaments $O$; maior $O-58$ sollicitud $O$ - 60 cambre $O$; fingint $O$; fatigave $O$ - 61 leixassen $O$; star $O$; anuiosa $O$; pus $O-62$ attenyer $O$; desijave $O$; lansant $O$; cors $O-64$ pensi $O$; leixant me $O$; viura $O$; fahia $O-66$ Encara me] encarem $O-67$ omicida $O-68$ enveyos $O$; cotinu $O$; vencer $O$; ésser] ser $O-69$ vensut $O$; ne $O$; legesa $O$; retraura $O-70$ granesa $O-71$ desigave $O$; spedient $O-73$ vinen $O-74$ accepte $O-75$ cors $O-76$ visca $O$; Sinarras $O$; pus $O-77$ enveyosos $O$; $\tan O-78$ derreres $O-79$ horelles $O$; perillave $O-81$ antigue $O$; temoros $O-83$ pererosament $O-84$ desliga $O-85$ terma $O$; acceptant $O-88$ vellesa $O-89$ tigra $O$; pietat $O-90$ dolras $O$; stranys $O$; belesa $O-92$ elegeixs $O-93$ temes $O$; sdevenidor $O-94$ deliure $O$; maior $O$; Lucrecia $O-95$ consentit $O-96$ sol $O-97$ aygue $O$; levara $O-98$ pus $O-99$ pensa $O-100$ descansar $O-101$ pus $O-102$ volras $O$; encare $O-103$ spers $O$ - 104 recomptar $O$; desespers $O$; succors $O-105$ vellesa $O$; stima $O$; de la anima $O-106$ offesa $O-107$ regna $O$; ho] o $O-108$ nigromancia $O-109$ pençes $O-111$ sta $O$; pus $O$; offici $O-113$ penses $O-116$ alegret $O$; extrema $O$; belesa $O-117$ plasents $O-118$ ta] te $O$; sespere $O-119$ stucia $O$; attenyer $O-120$ penses $O$; sdevenidors $O$; soferria $O-122$ furtunat quereles $O$; adversa $O$; sta $O-123$ regna $O-124$ hoynt $O-125$ un] hun $O$; tant $O$; suspir $O-126$ tamia $O$; reprencio $O-127$ axugant $O-130$ lançant-se] lensant se $O$; devant $O$ - 132 alegre $O-133 \mathrm{Mas}]$ mes $O$; se sdeve $O-135$ lenga $O-136$ inimiga $O-137$ consent $O-138$ horelles $O$ - 139 feresa $O-141$ querela $O-142$ envege $O$; acoste $O-143$ certa $O-144$ Sinnaras $O-145$ consenti $O$ 146 manifestave $O-147$ desorda $O$; aseiave $O-148$ deslimitat $O$; pus $O$; que lo] quel $O-149$ augmentave $O$; pensa $O$; speransa $O-151$ duptes $O$; desespers $O-152$ Sinarras $O$; faresa $O-153$ ia $O-154$ regna $O-155$ celebrar $O$; feste $O-156$ fructificave $O-157$ hornaven $O$; offertes $O$; llurs $O$; sacrifficis $O-159$ perague $O$ 159-160 fent-li] fahent li $O-161$ desplasia $O$; Sinarras $O-163$ descansaven $O$; ffebo $O$; occeano $O-164$ steles $O$; Tetis $O-165$ scilensi $O$; reposave $O-166$ sollicits $O$; enbaxada $O-167$ sperave $O-168$ tollia; e] y $O-169$ querelar $O$; anuiada $O-172$ satisfaccio $O-173$ tremontana $O-174$ cambre $O$; Sinarras $O-175$ alegre $O-176$ sta $O$; temorosa $O-178$ Sinarras $O-180$ concebuda $O$; brassos $O-181$ Sinarras $O$; sovint $O-182$ tamor $O$ - 183 sofferir $O$; sdevenidors $O-184$ laurora $O$; phebo $O$; embaxada $O-185$ Sinarras $O$ - 186 descansat $O$ 187 desigave $O-188$ legesa $O-190$ inimiga $O$; benvolensa $O-191$ esbeleyt $O$; felliren li $O-192 \tan ] \operatorname{tant} O$ - 194 fugir $O$; Sinarras $O$ - 195 puys $O$; speransa $O$; tingui $O$; grenea $O-196$ supliqui $O$; consentissen $O-197$ perquè los] perquels $O-198$ cors $O$; levada $O-199$ penitencia $O$; pus $O$; ensutzat $O$; ensutzas $O-200$ hoynt $O$; legesa $O$; benvolensa $O-201$ ésser] ser $O$; ho] o $O-202$ cors $O$; propri $O-203$ encara.s] encara se $O$; testifficant $O-206$ ho] o $O$; recomptar $O-207$ Narcisso $O-208$ continuu $O$; a la amargual ala margue $O-209$ abondantment $O$; enterrompent $O-210$ comensa $O-211$ Pus $O$; nostre $O$; desaguals $O$; anuigs $O-212$ attenyem $O$; recomptar $O-213$ scolten $O-214$ soffert $O-215$ querela $O-216$ extrema $O$; belesa $O-217$ beneventurada $O-219$ Pertint me $O$; enciosa $O$; edolescencia $O$; bellesa $O-220$ augmentave $O-221$ obeyr $O$ - 222 deffenia $O$; fletxes $O-223$ plasent $O$; aleujave $O$; anuigs $O-224$ Nimphes $O-225$ menyspresant $O$; bellesa $O-227$ querelave $O$; Nimpha $O$; Echo $O-228$ hoya $O-231$ vahent $O-232$ Echo $O-233$ pensa $O$; penitencia $O$; de ella $O$; prenguere $O$; venge $\mathrm{O}-234$ pus $O$; multitud $O-234-235$ d'aquí avant] de quiavant $O$ - 236 horelles $O-237$ continuu $O$; d'estrema] de extrema $O-238$ cremave $O$; podie $O$; bellesa $O$; lanamorave $O$ - 240 manifestave $O$; granesa $O-241$ lunyave $O-242$ meior $O$; rehons $O$; endressave $O-244$ pessave $O$; Nimpha $O$; sperant $O-246$ attenyia $O$; sguardar $O-247$ hun $O-248$ plasent $O$; scura $O$; hombra $O$; hun $O$ - 250 spil $O-251$ anuig $O$; extrema $O$; bellesa $O-252$ lenga $O$; Echo $O-253$ bellesa $O$; licencia $O-255$ extrema $O-266$ cremave $O-267$ sperar $O-268$ fugint $O-269$ lasparansa $O$; graciosa $O$; nimpha $O$; visque $O$ - 271 mentre $O$; visca $O-272$ mes $O$; pus $O$; lesperansa $O-273$ scures $O-274$ habitar $O-275$ cors $O-276$ eran $O-278$ se sguarden $O$; jutiave $O-279$ mes $O-282$ tant $O-283$ menyspresant $O-284$ extrem $O$; plasents $O-286$ diversos $O$; aucells $O$; alleuiave $O$; sollicits $O-288$ pessaven $O$; llur $O-289$ extrema $O$; phebo 
$O-290$ desemperant $O-292$ devall $O$; stave $O$; terre $O-293$ brodade $O$; diversitat $O$; regave $O-294$ plasent $O-295$ amonestave $O$; hoyen $O-296$ staven $O-297$ elegre $O$; mes $O-298$ portave $O$; genols $O-299$ assegi $O$; aygue $O$; Nimpha $O-300$ presencia $O$; tant $O-303$ graciosa $O-304$ offensa $O$; sperave $O-305$ lo stament $O-306$ comtemplave $O$; lo extrem $O-307$ bellesa $O$; benvolensa $O-310$ phebo $O$; fugir $O-311$ anuiosa $O$; plasent $O-312$ extrema $O$; bellesa $O-313$ haia $O$; menyspresat $O$; acceptes $O-314$ humils $O-316$ bellesa $O$; Narcisso $O-318$ offensa $O$; bellesa $O-319$ spere $O$; certa $O$; sols] sol $O-322$ sperave $O-323$ mostrave $O$; anuyar se $O$; rehonaven $O-324$ pensa $O$; soffaria $O-325$ separave $O$; rient $O$; mostrave $O-326$ plorave $O$; multitud $O$; lagrimes $O$; laygue $O$; clare $O-327$ augmentave $O-328$ horelles $O-329$ ho] o $O$; parlave $O-331$ delicade $O$; percebre $O$; asegi $O$ - 332 aygue $O$; tingui $O-333$ deesse $O$; pus $O$; mostrave $O$; acostave $O-334$ lavores $O$; mostrave $O$; tant $O$ - 335 anuyada $O$; continensa $O$; pus $O-336$ offensa $O$; reposave $O-337$ extrema $O$; attenyer $O-339$ pença $O$; encare $O-340$ anuiar $O-341$ rehonaven $O-342$ malvolensa $O$; mostrave $O$; extrem $O$; elegre $O-343$ bellesa $O-344$ aygue $O$; que los] quels $O-345$ Echo $O$; nimphes $O-346$ pus $O$; menyspresat $O-347$ tant $O-348$ viura $O$; ja.s] ja se $O$; narcisso $O-349$ orpha $O-350$ tant $O$; que.ls] que los $O$; phebo $O-351$ columpnes $O$; ercules $O-352$ pus $O-354$ ulls $O$; Nimpha $O-355$ anujave $O-356$ multitud $O$ - 357 lagrimes $O$; granesa $O$; extrema $O-358$ affany $O$; tant $O-360$ tant $O-361$ bellesa $O$; deffenien $O$ - 362 sperar $O-363$ comensi $O$; e] y $O-364$ carcer $O$; cors $O$; devellant $O-365$ pugue $O-369$ meior $O$; que als] quels $O$; stats $O-370$ beneventurats $O$; comparacio $O-371$ sobrepuie (con e finale scritta su a) $O-372$ llur $O$; furtuna $O$; vostros $O$; reho $O-373 \mathrm{sol} O-374$ manifestan $O$; sta $O-375$ trevessat $O$; tintes $O-376$ feresa $O$ - 377 piedosors $O$; recitadors $O$ - 378 manifestan $O$ - 379 consenten $O$; hoyts $O$; lagrimes $O$; volria $O$ - 380 scusar $O$; stimar $O-381$ propris $O-384$ ciutat $O$; hediffica $O-385$ deffenia $O-386$ que.ls] que los $O$; xichs $O$ - 387 attenyen $O$; Pyramus $O-388$ ho] o $O$; conaxença $O-390$ augmentant $O-391$ benvolensa $O-392$ contrestas $O$; crueltat $O-393$ asseiam $O$; licit $O$; pus $O-395$ deffania $O-396$ trobave $O-397$ atorgue $O$; Pyrramus $O$; Sinarras $O-398$ Nimpha $O$; Narcisso $O$; reho $O$; querelar me $O-399$ folament $O-400$ proha $O$; sculls $O-401$ fol $O$; pessatie $O-405$ stada $O-407$ care $O$; Pyramus $O-408$ veura $O$; ya $O$; de l'altra] dela altre $O$; sperave $O-409$ suspirant $O-410$ enveyosa $O$; deffens $O-411$ consents $O$; bocha $O$; se acosta $O-412$ desconegue $O-413$ pessatge $O-415$ samblants $O$; Pyramus $O$; tant $O$; delicade $O-416$ stil $O-417$ nostre $O$ - 418 sollicitud $O-419$ spay $O$; continuu $O-420$ extrema $O-421$ acustumades $O$; fingides $O-422$ llur $O$; studi $O$; Pyramus $O-424$ nostre $O-425$ lagrimes $O$; nostre $O-426$ Pyramus $O-427$ reparave $O$; ho] o $O$ - 428 resuscitaven $O$; reho $O-429$ vide $O-430$ augment $O$; de extrema $O-431$ sovint $O-433$ pensat $O$; plasent $O$; scures $O-434$ sollicitud $O$; fugint $O-435$ que lo] quel $O$; plasent $O$; aleuyant $O$; done $O-436$ certs $O$; d'esperar] de sperar $O$; hun $O-437$ ciutat $O$; sta $O-438$ venias $O-440$ hun $O$; spay $O$; Pyramus $O-446$ merevells $O$; forsat $O-447$ extrema $O$; sdevenidors $O-449$ sesguarden $O-450$ som $O$; cert $O-451$ anuiosa $O$ - 452 scura $O$; vaie $O-453$ ciutat $O-455$ a nostra] anostre $O-456$ inconvenients $O$; empetxar $O-457$ fugir $O-459$ tindre $O-461$ alcanse $O-463$ penses $O$; sdevenir $O-464$ pensar $O$; inconvenients $O-465$ atard $O$; attenyen $O$; flaquesa $O$; pensar $O-466$ cobre $O-469$ viage $O-470$ gracia $O-471$ anuies $O-473$ offira $O$ - 474 concert $O$; lagrimes $O$; pus $O-475$ licencia $O-476$ Pyramus $O$; phebo $O$; pariem $O-477$ cansats $O$; die $O$; maior $O$; spay $O-478$ acustumat $O-479$ detardave $O$; deffenia $O-480$ scura $O$; tard $O-481$ puys $O-484$ Pyramus $O-485$ speransa $O$; pyramus $O-487$ Pyramus $O$; arribat $O-488$ largue $O$; pensaments $O-489$ pensa $O$; conbatien $O-490$ stigui $O$; spay $O-492$ endressave $O$; fugir $O-493$ obscura $O-494$ hombre $O$; cove $O$; spay $O$; stave $O$; pensi $O-495$ anujat $O-499$ comptare $O$; Pyramus $O$; son $O-500$ compte $O$; é] he $O$ - 501 vingui $O-502$ ombre $O$; paraguem $O-504$ reluhia $O$ - 505 stant $O$; pensi $O-506$ altre $O$; vegade $O$; bistia $O$; cors $O-508$ stil $O$; ni] ne $O$; recitar $O$; granesa $O-509$ lansant me $O$; devant $O$; cors $O-511$ licencia $O$; alsa $O-512$ hun $O-513$ baxa $O$; forses $O-514$ persone $O-515 \tan$ gran] tant gran $O$; ya $O$; Phebo $O-516$ deurats $O$; comensave $O$; pyramus $O-517$ lexat $O$; fugint $O-518$ stave $O$; squinsat $O$; brave $O$; bistia $O-519$ augmenta $O$; granesa $O-521$ pugue $O$; fugir $O$; crueltat $O-522$ inplecables $O-524$ Pyramus $O-525$ hun $O$; spay $O-526$ vehesses $O$; desliure $O-527$ hun $O$; stalvi $O-528$ desliuraveu $O$; pus $O$; sabiau $O$; tan] tant $O$ - 529 approfite $O$; inreparable $O-530$ pus $O$; multitud $O$; resucitar $O-531$ strem $O$; anuiosa $O-532$ nostre $O$ - 533 nostre $O-534$ contrestat $O-535$ que.ls] que los $O$; cossors $O$; hun $O$ - 536 Encare $O$; lensi $O$; demunt $O$; spasa $O$; cors $O-537$ lexant $O$; brassos $O-538$ desaguals $O$; penas $O$.

\section{Acknowledgements}

The author wishes to thank the two anonymous reviewers for their careful reading of the paper and their useful suggestions. 


\section{Opere citate}

Agostinelli, Edvige; Coleman, William (ed.). 2015. Giovanni Boccaccio Teseida delle nozze di Emilia (Firenze: Edizioni del Galluzzo)

Ahuir i López, Artur (ed.). 1997. Les proses profanes de Joan Roüç de Corella (València: L’Oronella)

Alegre, Francesc. I494. 'Transformacions' del poeta Ouidi (Barcelona: Pere Miquel) < $\underline{\text { http:// }}$ $\underline{\text { tinyurl.com/zv3ld9c }>}$

Alemany Ferrer, Rafael. 20I5. 'Els elements d'articulació interna en les proses mitològiques de Joan Roís de Corella', in Studia mediaevalia Curt Wittlin dicata, ed. by Lola Badia, Emili Casanova, and Albert Hauf(Alacant: Institut Interuniversitari de Filologia Valenciana), pp. 33-5o <http:// tinyurl.com/zkb97zg>

Almiñana Vallés, Josep. 1984-1985. Obres de Joan Roiç de Corella, 2 vols.(València: Del Cénia al Segura)

Annicchiarico, Annamaria. 1996. Varianti corelliane e 'plagi' del 'Tirant': Achille e Polissena (Fasano di Brindisi: Schena Editore)

Annicchiarico, Annamaria. 2004. 'L'edizione critica delle Faules mitologiche di Joan Roís de Corella: bilanci, sondaggi, proposte', La Parola del Testo: Semestrale di Filologia e Letteratura Europea dalle Origini al Renascimento, 8 (=Studi in onore di Giuseppe E. Sansone, vol. 2): 44366

Annicchiarico, Annamaria. 20o6. 'Edizione e traduzione: Joan Roís de Corella', in La traduzione è una forma: trasmissione e sopravvivenza dei testi romanzi medievali, ed. by Giuseppina Brunetti and Gabriele Giannini, Quaderni di Filologia Romanza, 19: 57-75

Badia, Lola. I988. "'En les baixes antenes de vulgar poesia”: Corella, els mites i l'amor', nel suo De Bernat Metge a Joan Roís de Corella: estudis sobre la cultura literària de la tardor medieval catalana (Barcelona: Quaderns Crema), pp. I45-80 <http://tinyurl.com/htvlbe8 $>$

Badia, Lola. 1993. 'Per la presència d'Ovidi a l'Edat Mitjana catalana, amb notes sobre les traduccions de les Heroides i de les Metamorfosis al vulgar’, nel suo Tradició i modernitat als segles XIV i XV: estudis de cultura literària i lectures d'Ausiàs March (València: Institut Universitari de Filologia Valenciana; Barcelona: PAM), pp. 39-7I [prima in Studia in honorem prof. M. de Riquer, vol. I, Barcelona: Quaderns Crema, I986, pp. 79-Io9] < http://tinyurl.com/h756nqf>

Barchiesi, Alessandro (ed.). 2007. Ovidio Metamorfosi, vol. 2: Libri III-IV (Milano: Fondazione Lorenzo Valla; Mondadori)

Bassegoda Pineda, Enric. 20II. 'Vida i obra de Fra Bernat Hug de Rocabertí' (unpublished doctoral thesis, Universitat de Girona) <http://tinyurl.com/gl38ue7>

Bescós Prat, Pere. 20I4. 'Comentari i formació literària en Francesc Alegre: Ovidi i Bernardo Ilicino', Llengua \& Literatura, 24: 33-53 <http://dx.doi.org/10.2436/20.2502.01.67>

Bohigas, Pere; Soberanas, Amadeu J.; Espinàs, Noemí (ed.). 2005, Ausiàs March Poesies (Barcelona: Barcino)

Carbonell, Jordi (ed.). I973. Joan Roís de Corella Obres completes, I: Obra profana (València: Clàssics Albatros) 
Carrega, Annamaria. 20oI. 'La "sfera” di Narciso: riflessi di un mito sull'eros lirico medievale, L'immagine Riflessa (= Narciso, ed. by Andrea Calzolari), IO/I: 55-7I <http://tinyurl.com/ $\underline{\mathrm{hhmxsc} 3>}$

Chiner Gimeno, Jaume J. 20I4. 'Joan Roís de Corella, la seua vida i el seu entorn: noves dades per a la història de la cultura en la València del segle XV', Magnificat Cultura i Literatura Medievals, I: III-377 <http://dx.doi.org/10.7203/MCLM.1.3934>

Cingolani, Stefano M. 1997. 'D’Aquil.les a Jesús: reflexions sobre la cronologia de les obres de Joan Roís de Corella', Anuari de l'Agrupació Borrianenca de Cultura (= De literatura i cultura a la València medieval, ed. by Tomàs Martínez), 8: 67-85 <http://tinyurl.com/je6945a>

Cingolani, Stefano M. r998. Joan Roís de Corella: la importància de dir-se honest (València: Edicions 3 i 4 )

Delcorno, Carlo (ed.). r994. Giovanni Boccaccio Elegia di Madonna Fiammetta, in Giovanni Boccaccio Tutte le Opere, ed. by Vittore Branca (Milano: Mondadori)

Dombart, Bernhard; Kalb, Alfons (ed.). I955. Sancti Aurelii Augustini De civitate Dei, 2 vols. (Turnhout: Brepols)

Escartí, Vicent J. (ed.). 20I4. Joan Roís de Corella Obra completa (València: Institució Alfons el Magnànim; Diputació de València)

Fàbrega Escatllar, Valentí. 2004. 'El mite de Mirra en la versió de Roís de Corella', in Vestigia fabularum: la mitologia antiga a les literatures catalana i castellana entre l'edat mitjana i la moderna, ed. by Roger Friedlein and Sebastian Neumeister (Barcelona: Curial Ediciones Catalanes; PAM), pp. I79-89.

Gómez, Francesc J.; Pujol, Josep (ed.). 20o8. Ausiàs March Per haver d'amor vida: antologia comentada (Barcelona: Barcino)

Gómez, Francesc J. 20I5. 'Joan Roís de Corella: Proses d'inspiració clàssica i cortesa', in Història de la Literatura Catalana, vol. 3: Literatura Medieval III Segle XV, ed. by Lola Badia (Barcelona: Enciclopèdia Catalana; Barcino; Ajuntament de Barcelona), pp. 222-42

Gustà, Marina (ed.). I980o. Joan Roís de Corella Tragèdia de Caldesa i altres proses (Barcelona: Edicions 62; "la Caixa")

Hauf, Albert (ed.). 2005. Joanot Martorell (Martí Joan de Galba) Tirant lo Blanch (València: Tirant lo Blanch)

López Quiles, Antoni. 2007. Literatura i espiritualitat: aproximació a l’obra de Corella com a resposta davant Déu i l'home (València: Institució Alfons el Magnànim)

López Quiles, Antoni. 20I4. 'La proposta sapiencial de Corella', in Joan Roís de Corella i el seu món (València: Institució Alfons el Magnànim), pp. 389-422

López Quiles, Antoni; Puig Gisbert, Abel. 20I4. Ensenyar, commoure i adelitar: notes de propedèutica per a rellegir Corella (Catarroja: Perifèric Edicions)

Mancini, Mario (ed.). 1989. Il lai di Narciso (Parma: Pratiche Editrice)

Martí, Sadurní. I997. 'El Cançoner del marquès de Barberà (S $\mathrm{S} / \mathrm{BMI})$ : descripció codicològica', Boletín Bibliográfico de la Asociación Hispánica de Literatura Medieval, iг: 463-502 <http:// tinyurl.com/zdutc2r> 
Martínez, Tomàs. 2OII. 'Joan Roís de Corella i la literatura a la València de la segona meitat del Xv', in Panorama crític de la literatura catalana, vol. 2: Edat mitjana: Segle d'or, ed. by Albert Hauf (Barcelona: Vicens Vives), pp. 435-77

Martínez, Tomàs. 20I3. L'obra literària de Joan Roís de Corella, in Biblioteca Virtual Miguel de Cervantes-Joan Lluís Vives <http://tinyurl.com/pxg2wyq> [accessed 28-O5-20I6]

Martos, Josep Ll. (ed.). 2oora. Les proses mitològiques de Joan Roís de Corella: edició crítica, (Alacant: Institut Interuniversitari de Filologia Valenciana; Barcelona: PAM) < $\underline{\text { http://tinyurl. }}$ $\underline{\mathrm{com} / \mathrm{zm} 3 \mathrm{qx} 3 \mathrm{r}>}$

Martos, Josep L1. 20oIb. Fonts i seqüència cronològica de les proses mitològiques de Joan Roís de Corella (Universitat d'Alacant: Departament de Filologia Catalana)

Martos, Josep L1. 2002. 'Los espacios en blanco y la estructura del Cançoner del marqués de Barberä, in Proceedings of the Eleventh Colloquium of the MHRS, ed. by Alan Deyermond and Jane Whetnall, PMHRS, 34 (London: Queen Mary University of London), pp. 57-65 <http:// tinyurl.com/z5j82b4>

Miquel i Planas, Ramon (ed.). r9ı3. Obres de J. Roiç de Corella (Barcelona: Biblioteca Catalana)

Miralles, Carles. 1978. 'Raons de Mirra en boca d'Esperança: sobre un altre plagi de Roís de Corella en el Tirant lo Blanc', Boletín de la Real Academia de Buenas Letras de Barcelona, 37: I4I-47 [poi nel suo Eulàlia: estudis inotes de literatura catalana (Barcelona: Edicions del Mall, I986,


Interuniversitari de Filologia Valenciana; Barcelona: PAM, 2OI2, pp. I59-65] <http://tinyurl. $\underline{\text { com/jld7n } 5 \mathrm{~b}>}$

Miralles, Carles. 199r. 'Raons de Mirra en boca de Carmesina: encara un altre plagi de Roís de Corella en el Tirant lo Blanc', in Estudis de Llengua i Literatura Catalanes, vol. 23 (=Miscel-lània Jordi Carbonell vol. 2), (Barcelona: PAM), pp. 5-16 [poi nel suo Aracne. Trasllats i ordits d'alguns textos del Quatre-cents, València: Institut Interuniversitari de Filologia Valenciana; Barcelona: PAM, 2OI2, pp. I67-76] <http://tinyurl.com/hvogh3n>

Miralles, Carles. 1998. 'Corella i el Tirant: qüestions d'intertextualitat', Caplletra, 24: 67-79 [poi nel suo Aracne. Trasllats i ordits d'alguns textos del Quatre-cents, València: Institut Interuniversitari de Filologia Valenciana; Barcelona: PAM, 20I2, pp. I77-93) <http://tinyurl.com/gwkbtbc $>$

Moreschini, Claudio (ed.). 20oo. Boethius Anicius Manlius Severinus De consolatione philosophiae (Monaco: K.G. Saur)

Pelai Briz, Francesc (ed.). I868. 'Lamentacions de Mirra e Narciso e Tisbe per mossen Corella cavaller e en sacra theologia professor', in Llibre intitolat "Jardinet d'orats" (Barcelona: Joan Roca y Bros) pp. 95-III

Pellisa Prades, Gemma. 20I3. La ficció sentimental catalana de la segona meitat del segle XV (unpublished doctoral thesis, Universitat de Barcelona) $<\underline{\text { http://tinyurl.com/hpa2f8m }>}$

Pujol, Josep. 2002. La memòria literària de Joanot Martorell: models i escriptura en el Tirant lo Blanc (Barcelona: Curial Edicions Catalanes; PAM)

Pujol, Josep. 20I2. 'Les traduccions hispàniques de les Heroides d'Ovidi i els Bursarï Ovidianorum de Guillem d'Orléans: una aproximació', in Estudios de latín medieval hispánico: V Congreso Internacional de Latín Medieval Hispánico, ed. by José Martínez Gázquez, Óscar de la Cruz Palma, and Cándida Ferrero Hernández (Firenze: SISMEL; Edizioni del Galluzzo), pp. 245-58 
Pujol, Josep. 20I4. 'Para las fuentes del Bursario: la traducción catalana de las Heroidas de Guillem Nicolau y su versión castellana anónima', Bulletin of Hispanic Studies, 9I: $453-76<$ http://dx.doi. org/10.3828/bhs.2014.29>

Reed, Joseph D. (ed.). 20I3. Ovidio Metamorfosi, vol. 5: Libri X-XII(Milano: Fondazione Lorenzo Valla; Mondadori)

Riquer, Martí de. I964. Història de la literatura catalana: part antiga, 3 vols. (Barcelona: Ariel)

Soler, Abel; Navarro, Miquel; Pons, Vicent. 20I4. Joan Roís de Corella (1435-1497): síntesi biogràfica i aportació documental (València: Acadèmia Valenciana de la Llengua)

Thiry-Stassin, Martine; Tyssens, Madeleine (ed.). 1976, Narcisse: conte ovidien français du XII siècle (Paris: Les Belles Lettres) <http://tinyurl.com/jtjod5m $>$

Tosi, Renzo. I993. Dizionario delle sentenze latine e greche, $9^{\text {a }}$ ed. (Milano: Rizzoli)

Turró, Jaume. 1992. 'El ms. I5I de la Biblioteca Universitària de Barcelona (Jardinet de orats): descripció i estudi codicològic', Boletín Bibliográfico de la Asociación Hispánica de Literatura Medieval, 6/I: I-55

Turró, Jaume, I996. 'El mite de Caldesa: Corella al Jardinet d'orats', Atalaya: Révue Française d'Études Médiévales Hispaniques, 7: IO3-16 <http://tinyurl.com/h8acs5j> [accessed 28-O52OI6]

Verger, Eduard. 20II. 'Sotsmissió amorosa de Roís de Corella, amb una postil.la', in Estudis de Llengua i Literatura Catalanes, vol. 62 (=Miscel-lània Albert Hauf I), (Barcelona: PAM), pp. 57${ }_{7} 6<$ http://tinyurl.com/jd3djbl $>$ [checked 28-05-20I6]

Villar, Milagros. I995. Códices petrarquescos en España (Padova: Editrice Antenore)

Walther, Hans. 1982-1986. Proverbia sententiaeque Latinitatis Mediu ac Recentioris Aevi. Nova Series: Lateinische Sprichwörter und Sentenzen des Mittelalters und der frühen Neuzeit in alphabetischer Anordnung, ed. by Paul Gerhard Schmidt, 3 vols. (Göttingen: Vandenhoeck \& Ruprecht)

Wittlin, Curt. 1997. 'La Biblis, Mirra i Santa Maria de Joan Roís de Corella: traduccions modulades, amplificades i adaptades', in Anuari de l'Agrupació Borrianenca de Cultura (=De literatura i cultura a la València medieval, ed. by Tomàs Martínez), 8: 175-89 <http://tinyurl.com/ g15uf2a>

Zaccaria, Vittorio (ed.). 1998. Giovanni Boccaccio Genealogie deorum gentilium, in Giovanni Boccaccio, Tutte le Opere, ed. by Vittore Branca (Milano: Mondadori) 